\begin{tabular}{|c|l|}
\hline Title & Global dynamics of a stochastic neuronal oscillator \\
\hline Author(s) & Yamanobe, Takanobu \\
\hline Citation & $\begin{array}{l}\text { Physical Review E, 88(5), 052709_1-052709-19 } \\
\text { https://doi.org/10.1103/PhysRevE.88.052709 }\end{array}$ \\
\hline Issue Date & 2013-11 \\
\hline Doc URL & http://hdl.handle.net/2115/53513 \\
\hline Rights & Copyright $\odot$ 2013A merican Physical Society \\
\hline Type & article \\
\hline File Information & PHYSICAL REVIEW E 88_052709.pdf \\
\hline
\end{tabular}

Instructions for use 


\title{
Global dynamics of a stochastic neuronal oscillator
}

\author{
Takanobu Yamanobe* \\ Hokkaido University School of Medicine, North 15, West 7, Kita-ku, Sapporo 060-8638, Japan \\ and PRESTO, Japan Science and Technology Agency (JST), 4-1-8 Honcho Kawaguchi, Saitama 332-0012, Japan
}

(Received 5 August 2013; published 13 November 2013)

\begin{abstract}
Nonlinear oscillators have been used to model neurons that fire periodically in the absence of input. These oscillators, which are called neuronal oscillators, share some common response structures with other biological oscillations such as cardiac cells. In this study, we analyze the dependence of the global dynamics of an impulse-driven stochastic neuronal oscillator on the relaxation rate to the limit cycle, the strength of the intrinsic noise, and the impulsive input parameters. To do this, we use a Markov operator that both reflects the density evolution of the oscillator and is an extension of the phase transition curve, which describes the phase shift due to a single isolated impulse. Previously, we derived the Markov operator for the finite relaxation rate that describes the dynamics of the entire phase plane. Here, we construct a Markov operator for the infinite relaxation rate that describes the stochastic dynamics restricted to the limit cycle. In both cases, the response of the stochastic neuronal oscillator to time-varying impulses is described by a product of Markov operators. Furthermore, we calculate the number of spikes between two consecutive impulses to relate the dynamics of the oscillator to the number of spikes per unit time and the interspike interval density. Specifically, we analyze the dynamics of the number of spikes per unit time based on the properties of the Markov operators. Each Markov operator can be decomposed into stationary and transient components based on the properties of the eigenvalues and eigenfunctions. This allows us to evaluate the difference in the number of spikes per unit time between the stationary and transient responses of the oscillator, which we show to be based on the dependence of the oscillator on past activity. Our analysis shows how the duration of the past neuronal activity depends on the relaxation rate, the noise strength, and the impulsive input parameters.
\end{abstract}

DOI: 10.1103/PhysRevE.88.052709

PACS number(s): 87.19.1c, 87.19.1s, 02.50.Fz, 05.10.Gg

\section{INTRODUCTION}

In nervous systems, information is transmitted via spikes; however, the question of whether information is carried via detailed spike patterns (temporal or timing coding) or simply by the number of spikes in a given time period (rate coding) is a subject of active debate [1]. Each neuron in a neural network receives inputs from other neurons or outside of the neural network and transforms the inputs into spikes based on the intrinsic dynamics of the neuron. In the theory of artificial neural networks, the inputs are transformed by a function (for example, sigmoid function) and information carrier in an artificial neural network depends on the selection of the function. Thus, it is important to investigate how each neuron transforms the inputs into spikes. One necessary condition for temporal coding is that the spike generation of a neuron must not depend substantially on the past spike generation. Thus, the duration of the transient regime in neuronal activity must be short enough to achieve this independence. This means that the properties of the transient regime of the neuronal activity may then offer insights into whether the pattern of spikes is a possible information carrier in nervous systems. Because information processing in nervous systems may occur in the transient regime, the transient dynamics of neurons and neuronal models [2-5], and those of neural networks [6,7], are the focus of analysis.

Nonlinear oscillators have been used to model neurons that fire periodically in the absence of input [8,9]. These nonlinear oscillators are called neuronal oscillator and are

*yamanobe@med.hokudai.ac.jp a subclass of nonlinear oscillators that are also found in a wide variety of biological and complex systems such as cardiac cells [10,11], respiratory rhythm generation [12,13], Josephson junctions [14], and climate dynamics [15]. In biological systems, the system may converge slowly to the asymptotic dynamics. In this case, the transient dynamics of the system can occur far from the asymptotic structure. Thus, it is important to analyze the global dynamics of the nonlinear oscillators to understand the transient dynamics. However, as the global dynamics are difficult to understand, higher-order dynamics are usually analyzed after a reduction to lower-order dynamics. The most successful approach has been the phase reduction method $[16,17]$. If a nonlinear oscillator is weakly perturbed, the trajectories will be in the neighborhood of the limit cycle of the nonlinear oscillator. Thus, the dynamics can be approximated by a vector field on the limit cycle, and this makes it possible to represent the limit-cycle dynamics in a higher-dimensional phase space by a one-dimensional (1D) variable called the phase.

The impulse-driven nonlinear oscillators are often used in the analysis of neuronal or biological oscillators [4,17-25], and depending on the relaxation rate to the limit cycle, the state points of the nonlinear oscillator can be far from the limit cycle. For example, Glass and Sun [20] analyzed the dependence of the bifurcation structure of an impulse-driven nonlinear oscillator on the relaxation rate. For their analysis, they used a 2D extension of the phase transition curve for the nonlinear oscillator, which represents the phase shift due to a single isolated impulse. However, it is usually only the dynamics in the asymptotic regime that are analyzed since there are few available tools for analyzing the dynamics in the transient regime. 
Noise will also affect the dynamics, since intrinsic noise, e.g., ion channel noise [26], might restrict the accuracy of the spike generation. In general, nonlinear systems are often influenced by stochastic fluctuations. In our previous study, we introduced a Markov operator for an impulse-driven stochastic neuronal oscillator that can approximate the density evolution in the entire phase space of the oscillator driven by timevarying impulses [5].

In this paper, we analyze the global dynamics of a stochastic neuronal oscillator driven by time-varying impulses by changing the relaxation rate to the limit cycle, intrinsic noise strength, and input impulse parameters. We introduce a Markov operator for an infinite relaxation rate using the small disturbance asymptotic theory $[27,28]$, and this operator describes the stochastic dynamics around the limit cycle. We investigate the dynamics of the entire phase space without input impulses using our Markov operator for finite and infinite relaxation rates and analyze the response of the stochastic neuronal oscillator to impulsive inputs by examining the effects of the relaxation rate, intrinsic noise strength, and input impulse parameters. For both the finite and infinite cases, the response of the stochastic neuronal oscillator to time-varying impulses is described by the product of the Markov operators. We can decompose the Markov operator into stationary and transient components based on the properties of eigenvalues and eigenfunctions to identify the components that affect the current response. Moreover, we introduce a stochastic rotation number to relate the dynamics of the oscillator to the number of spikes per unit time and the interspike interval (ISI) density to understand the steady state dynamics of the oscillator. Specifically, we analyze the components of the stochastic rotation number based on the properties of the Markov operator. In relation to the information carrier in nervous systems, we show how the past activity of the stochastic neuronal oscillator affects the current firing rate. We demonstrate that there can exist the long-range dependence of the current neuronal activity on the past activity depending on the relaxation rate, the noise strength, and input parameters.

\section{METHODS}

\section{A. Stochastic Poincaré oscillator}

The Poincaré oscillator and its variant are a member of a set of systems that are widely used in the analysis of neuronal or biological oscillators [4,9,17-24,29-31]. Based on [5,20], we introduce the Poincaré oscillator here and summarize its properties. The oscillator can be described in polar coordinates as

$$
\frac{d R_{t}^{(0)}}{d t}=K R_{t}^{(0)}\left(1-R_{t}^{(0)}\right), \quad \frac{d \Phi_{t}^{(0)}}{d t}=1,
$$

where $R_{t}^{(0)} \in\{x ; x>0, x \in \mathbb{R}\}$ is the radial coordinate, and $\Phi_{t}^{(0)} \in S^{1}\left(S^{1}\right.$ is the unit circle) is the normalized angular coordinate that varies in $[0,1)$; the superscript $(0)$ indicates the deterministic case and the subscript represents the time $t$. The positive parameter $K$ is the relaxation rate to the limit cycle; the stable limit cycle is the unit circle with period 1. Trajectories starting from any initial point in the phase plane, except the origin, wind counterclockwise around the origin and converge to the limit cycle as $t \rightarrow \infty$. We define $X_{t}^{(0)}=R_{t}^{(0)} \cos \left(2 \pi \Phi_{t}^{(0)}\right)$ as the "membrane potential" and $Y_{t}^{(0)}=R_{t}^{(0)} \sin \left(2 \pi \Phi_{t}^{(0)}\right)$ as the "refractoriness." The spike occurs when the state point crosses the positive $x$ axis (For the detailed reasons to use the Poincaré oscillator, see the Appendix.) We consider the relationship between the state point just before the $n$th impulse and that just before the $(n+$ 1)th impulse. Following [20], we define the $n$th impulsive stimulation by an instantaneous horizontal shift by an amount $A_{n}$, where $n$ denotes the $n$th impulse. If an impulse with amplitude $A_{n}$ shifts a state point $\left(r_{n}, \phi_{n}\right)$ to the point $\left(r_{n}^{\prime}, \phi_{n}^{\prime}\right)$, the relation becomes

$$
\begin{aligned}
r_{n}^{\prime} & =F_{R}\left(r_{n}, \phi_{n}\right)=\left[r_{n}^{2}+A_{n}^{2}+2 A_{n} r_{n} \cos \left(2 \pi \phi_{n}\right)\right]^{1 / 2}, \\
\phi_{n}^{\prime}=F_{\Phi}\left(r_{n}, \phi_{n}\right) & =\frac{1}{2 \pi} \arccos \frac{r_{n} \cos \left(2 \pi \phi_{n}\right)+A_{n}}{F_{R}\left(r_{n}, \phi_{n}\right)},
\end{aligned}
$$

where the subscripts $R$ and $\Phi$ denote the shift in the directions of the radial and normalized angular coordinates, respectively. To evaluate the arc-cosine function, we should take $0<\phi_{n}^{\prime}<$ 0.5 for $0<\phi_{n}<0.5$ and $0.5<\phi_{n}^{\prime}<1$ for $0.5<\phi_{n}<1$. Equation (2) is the 2D version of the phase transition curve of this oscillator, which represents the phase shift due to a single isolated impulse.

After the $n$th interimpulse interval $I_{n}$, the state point starting from the initial point $\left(r_{n}^{\prime}, \phi_{n}^{\prime}\right)$, as determined by Eq. (1), is expressed as follows:

$$
\begin{aligned}
r_{n+1} & =R_{I_{n}}^{(0)}=r_{n}^{\prime} /\left\{\left(1-r_{n}^{\prime}\right) e^{-K I_{n}}+r_{n}^{\prime}\right\}, \\
\phi_{n+1} & =\Phi_{I_{n}}^{(0)}=\phi_{n}^{\prime}+I_{n} \quad(\bmod 1) .
\end{aligned}
$$

In the case of $K \rightarrow \infty$, the dynamics of the oscillator are described only by the normalized angular coordinate. Thus, the effect of the $n$th impulse is

$$
\phi_{n}^{\prime}=\tilde{F}_{\Phi}\left(\phi_{n}\right)=\frac{1}{2 \pi} \arccos \frac{\cos \left(2 \pi \phi_{n}\right)+A_{n}}{\sqrt{1+A_{n}^{2}+2 A_{n} \cos \left(2 \pi \phi_{n}\right)}},
$$

which defines the phase transition curve for this model. $\tilde{F}_{\Phi}\left(\phi_{n}\right)-\phi_{n}$ corresponds to the phase response curve, which shows the phase shift due to an impulse, and the shape of the phase response curve is biphasic if $|A|<1$ [9]. The biphasic phase response curves are observed experimentally (for example, [32]). The tilde denotes the function as $K \rightarrow \infty$. In what follows, we use the tilde for functions and variables when it is necessary to indicate that $K \rightarrow \infty$. Using this phase transition curve, the state point just before the $(n+1)$ th impulse becomes

$$
\phi_{n+1}=\tilde{\Phi}_{I_{n}}^{(0)}=\phi_{n}^{\prime}+I_{n} \quad(\bmod 1),
$$

where $\phi_{n}^{\prime}=\tilde{F}_{\Phi}\left(\phi_{n}\right)$. In previous study, we transform Eq. (1) into Cartesian coordinates and include a noise term in the expression for the membrane potential. In polar coordinates, the Poincaré oscillator with the noise term can be expressed 
as [5]

$$
\begin{aligned}
d R_{t}^{(\epsilon)}= & K R_{t}^{(\epsilon)}\left(1-R_{t}^{(\epsilon)}\right) d t+\frac{\epsilon^{2}}{2} \frac{\sin ^{2}\left(2 \pi \Phi_{t}^{(\epsilon)}\right)}{R_{t}^{(\epsilon)}} d t \\
& +\epsilon \cos \left(2 \pi \Phi_{t}^{(\epsilon)}\right) d W_{t}, \\
d \Phi_{t}^{(\epsilon)}= & d t+\frac{\epsilon^{2}}{4 \pi} \frac{\sin \left(4 \pi \Phi_{t}^{(\epsilon)}\right)}{R_{t}^{(\epsilon)}} d t-\frac{\epsilon}{2 \pi} \frac{\sin \left(2 \pi \Phi_{t}^{(\epsilon)}\right)}{R_{t}^{(\epsilon)}} d W_{t},
\end{aligned}
$$

where the superscript indicates the dependence of the random variables on the strength of the noise term $\epsilon \in(0,1]$, for which $\epsilon=0$ gives the deterministic case. The 1D standard Wiener process is denoted by $W_{t}$. We refer to Eq. (6) as a stochastic Poincaré oscillator.

\section{B. Stochastic phase transition operator}

In our previous study [5], we introduced a Markov operator that relates the density of the state points just before the $n$th impulse to that just before the $(n+1)$ th impulse. We called this Markov operator a stochastic phase transition operator (SPTO). In what follows, we derive SPTOs for infinite $K$. According to Eq. (2), the state point just before the $n$th impulse $\left(r_{n}, \phi_{n}\right)$ shifts to $\left(r_{n}^{\prime}, \phi_{n}^{\prime}\right)$ after the $n$th impulse. After this shift, the dynamics of the state point are described by the following integral equation with the initial condition $\left(r_{n}^{\prime}, \phi_{n}^{\prime}\right)$ defined by Eq. (2):

$$
\begin{aligned}
R_{I_{n}}^{(\epsilon)}= & r_{n}^{\prime}+K \int_{0}^{I_{n}} R_{s}^{(\epsilon)}\left(1-R_{s}^{(\epsilon)}\right) d s \\
& +\frac{\epsilon^{2}}{2} \int_{0}^{I_{n}} \frac{\sin ^{2}\left(2 \pi \Phi_{s}^{(\epsilon)}\right)}{R_{s}^{(\epsilon)}} d s+\epsilon \int_{0}^{I_{n}} \cos \left(2 \pi \Phi_{s}^{(\epsilon)}\right) d W_{s} \\
\Phi_{I_{n}}^{(\epsilon)}= & \phi_{n}^{\prime}+I_{n}+\frac{\epsilon^{2}}{4 \pi} \int_{0}^{I_{n}} \frac{\sin \left(4 \pi \Phi_{s}^{(\epsilon)}\right)}{R_{s}^{(\epsilon)^{2}}} d s \\
& -\frac{\epsilon}{2 \pi} \int_{0}^{I_{n}} \frac{\sin \left(2 \pi \Phi_{s}^{(\epsilon)}\right)}{R_{s}^{(\epsilon)}} d W_{s}, \quad(\bmod 1)
\end{aligned}
$$

In the case of $K \rightarrow \infty$, the dynamics of the stochastic Poincaré oscillator can be described by the dynamics on the limit cycle. As $K \rightarrow \infty, R_{I_{n}}^{(\epsilon)}=1$ and the dynamics of $\Phi_{t}^{(\epsilon)}$ are given by

$$
\begin{aligned}
\Phi_{t}^{(\epsilon)}= & \tilde{\Phi}_{I_{n}}^{(\epsilon)}=\phi_{n}^{\prime}+I_{n}+\frac{\epsilon^{2}}{4 \pi} \int_{0}^{I_{n}} \sin \left(4 \pi \tilde{\Phi}_{s}^{(\epsilon)}\right) d s \\
& -\frac{\epsilon}{2 \pi} \int_{0}^{I_{n}} \sin \left(2 \pi \tilde{\Phi}_{s}^{(\epsilon)}\right) d W_{s}, \quad(\bmod 1),
\end{aligned}
$$

where $\phi_{n}^{\prime}$ is defined by Eq. (4). We call Eq. (8) a phase equation and note that it includes the modification term suggested by Yoshimura and Arai [33] since we take $K \rightarrow \infty$ after the coordinate transform.

We introduce a new random variable $\Theta_{t}^{(\epsilon)}=\Phi_{t}^{(\epsilon)}(\bmod 1)$ that takes a value in $\mathbb{R}$ and explicitly indicates the rotation around the origin. Equation (7) then becomes

$$
\begin{aligned}
R_{I_{n}}^{(\epsilon)}= & r_{n}^{\prime}+K \int_{0}^{I_{n}} R_{s}^{(\epsilon)}\left(1-R_{s}^{(\epsilon)}\right) d s \\
& +\frac{\epsilon^{2}}{2} \int_{0}^{I_{n}} \frac{\sin ^{2}\left(2 \pi \Theta_{s}^{(\epsilon)}\right)}{R_{s}^{(\epsilon)}} d s+\epsilon \int_{0}^{I_{n}} \cos \left(2 \pi \Theta_{s}^{(\epsilon)}\right) d W_{s},
\end{aligned}
$$

$$
\begin{aligned}
\Theta_{I_{n}}^{(\epsilon)}= & \phi_{n}^{\prime}+I_{n}+\frac{\epsilon^{2}}{4 \pi} \int_{0}^{I_{n}} \frac{\sin \left(4 \pi \Theta_{s}^{(\epsilon)}\right)}{R_{s}^{(\epsilon)^{2}}} d s \\
& -\frac{\epsilon}{2 \pi} \int_{0}^{I_{n}} \frac{\sin \left(2 \pi \Theta_{s}^{(\epsilon)}\right)}{R_{s}^{(\epsilon)}} d W_{s} .
\end{aligned}
$$

Similarly, for Eq. (8), we introduce the random variable $\widetilde{\Theta}_{I_{n}}^{(\epsilon)}=$ $\tilde{\Phi}_{I_{n}}^{(\epsilon)}(\bmod 1)$, and Eq. (8) becomes

$$
\begin{aligned}
\tilde{\Theta}_{I_{n}}^{(\epsilon)}= & \phi_{n}^{\prime}+I_{n}+\frac{\epsilon^{2}}{4 \pi} \int_{0}^{I_{n}} \sin \left(4 \pi \tilde{\Theta}_{s}^{(\epsilon)}\right) d s \\
& -\frac{\epsilon}{2 \pi} \int_{0}^{I_{n}} \sin \left(2 \pi \tilde{\Theta}_{s}^{(\epsilon)}\right) d W_{s} .
\end{aligned}
$$

In what follows, we call $\Theta_{I_{n}}^{(\epsilon)}$ and $\tilde{\Theta}_{I_{n}}^{(\epsilon)}$ a lifted angular coordinate. To calculate the stochastic kernels of the SPTO, i.e., the transition density corresponding to a given stochastic differential equation, we apply the small disturbance asymptotic theory, which is an asymptotic expansion of the stochastic processes $[27,28]$. To apply this theory, we assume that the diffusion coefficients in Eqs. (9) and (10) are not zero for any $s>0$. This assumption guarantees the asymptotic expansion of the transition density around the normal distribution density. Thus, we have to apply the theory to Eqs. (9) and (10) separately to calculate the transition density. We derived the stochastic kernel for the full equation [Eq. (9)] in our previous study [5]. Here, we derive the stochastic kernel of the SPTO for Eq. (10). We first expand $\tilde{\Theta}_{t}^{(\epsilon)}$ with respect to $\epsilon$ as

$$
\tilde{\Theta}_{I_{n}}^{(\epsilon)}=\tilde{\Theta}_{I_{n}}^{(0)}+\epsilon A_{1 \tilde{\Theta} I_{n}}+o(\epsilon),
$$

where $\tilde{\Theta}_{I_{n}}^{(0)}$ is a deterministic solution of Eq. (10) and $A_{1 \tilde{\Theta} I_{n}}=$ $\left.\frac{\partial \tilde{\Theta}_{I_{n}}^{(\epsilon)}}{\partial \epsilon}\right|_{\epsilon=0}$, where the subscript $\tilde{\Theta} I_{n}$ denotes the derivative of $\tilde{\Theta}_{I_{n}}^{(\epsilon)}$ explicitly. The derivative is

$$
A_{1 \tilde{\Theta} I_{n}}=-\frac{1}{2 \pi} \int_{0}^{I_{n}} \sin \left(2 \pi \tilde{\Theta}_{s}^{(0)}\right) d W_{s} .
$$

To consider the stochastic dynamics around the deterministic solution $\tilde{\Theta}_{I_{n}}^{(0)}$, we introduce a new random variable $\tilde{S}_{I_{n}}^{(\epsilon)}=$ $\left(\tilde{\Theta}_{I_{n}}^{(\epsilon)}-\tilde{\Theta}_{I_{n}}^{(0)}\right) / \epsilon$. The expansion of $\tilde{S}_{I_{n}}^{(\epsilon)}$ with respect to $\epsilon$ gives

$$
\tilde{S}_{I_{n}}^{(\epsilon)}=A_{1 \tilde{\Theta} I_{n}}+o(1)
$$

The asymptotic expansion of the characteristic function of $\tilde{S}_{I_{n}}^{(\epsilon)}$ with respect to $\epsilon$ is

$$
\begin{aligned}
\Psi(\xi) & =E\left[\exp \left\{i \xi\left(A_{1 \tilde{\Theta} I_{n}}+o(1)\right)\right\}\right] \\
& =E\left[\exp \left(i \xi A_{1 \tilde{\Theta} I_{n}}\right)\{1+o(1)\}\right] \\
& =E\left[\exp \left(i \xi A_{1 \tilde{\Theta} I_{n}}\right)\right]+o(1) \\
& =\exp \left\{-\frac{1}{2}\left(\Sigma_{\tilde{\Theta} I_{n}}\left(\phi_{n}\right) \xi^{2}\right)\right\}+o(1),
\end{aligned}
$$

where $\xi \in \mathbb{R} \quad$ and $\Sigma_{\tilde{\Theta} I_{n}}\left(\phi_{n}\right)=E\left[A_{1 \tilde{\Theta} I_{n}}^{2}\right]=\left(\frac{1}{2 \pi}\right)^{3}\left\{\pi I_{n}-\right.$ $\left.\frac{1}{2} \cos \left[2 \pi\left(2 \tilde{F}_{\Phi}\left(\phi_{n}\right)+I_{n}\right)\right] \sin \left(2 \pi I_{n}\right)\right\}$. In Eq. (11), the second equality is derived from the expansion of the exponential function with respect to $\epsilon$. The fourth equality is derived from the Gaussianity of $A_{1 \tilde{\Theta} I_{n}}$. Using the inverse Fourier transform of Eq. (11), we obtain

$$
f_{\tilde{S}_{I_{n}}^{(\epsilon)}}(s)=n\left[s ; 0, \Sigma_{\tilde{\Theta} I_{n}}\left(\phi_{n}\right)\right]+o(1)
$$


where $s \in \mathbb{R}$ and $n\left[s ; 0, \Sigma_{\tilde{\Theta}_{I_{n}}}\left(\phi_{n}\right)\right]$ is the density of a $1 \mathrm{D}$ Gaussian distribution with zero mean and a variance of $\Sigma_{\tilde{\Theta} I_{n}}\left(\phi_{n}\right)$; the subscript denotes the density of the random variable $\tilde{S}_{I_{n}}^{(\epsilon)}$. Equation (12) is derived by expanding around the solution $\tilde{\Theta}_{I_{n}}^{(0)}$, and the obtained density is the 1D Gaussian distribution in the direction of $\tilde{\Theta}$. We use Eq. (12) to approximate the density of $\tilde{\Theta}_{I_{n}}^{(\epsilon)}$ as

$$
f_{\tilde{\Theta}_{I_{n}}^{(\epsilon)}}\left(\theta ; \phi_{n}\right) \simeq n\left[\theta ; \tilde{\Theta}_{I_{n}}^{(0)}\left(\phi_{n}\right), \epsilon^{2} \Sigma_{\tilde{\Theta}_{I_{n}}}\left(\phi_{n}\right)\right],
$$

where $s=\left(\theta-\Theta_{I_{n}}^{(0)}\right) / \epsilon$. The dependence on $\phi_{n}$ is explicit in the term $\tilde{\Theta}_{I_{n}}^{(0)}\left(\phi_{n}\right)$, and the function $n\left[\theta ; \tilde{\Theta}_{I_{n}}^{(0)}\left(\phi_{n}\right), \epsilon^{2} \Sigma_{\tilde{\Theta}_{I_{n}}}\left(\phi_{n}\right)\right]$ depends on $\phi_{n}$ via Eqs. (4) and (5). In this way, the difficulty of the discontinuity caused by the impulse is avoided when approximating $f_{\tilde{\Theta}_{I_{n}}^{(\epsilon)}}\left(\theta ; \phi_{n}\right)$. Using Eq. (13), the stochastic kernel for the phase equation is given by

$$
g_{\infty, \epsilon, A_{n}, I_{n}}\left(\phi ; \phi_{n}\right)=\sum_{p=-\infty}^{p=+\infty} n\left[\phi+p ; \Theta_{I_{n}}^{(0)}\left(\phi_{n}\right), \epsilon^{2} \Sigma_{\tilde{\Theta}_{I_{n}}}\left(\phi_{n}\right)\right],
$$

where $\phi=\theta(\bmod 1)$. The summation with respect to $\phi$ takes into account multiple rotations around the origin, and the dependence of the stochastic kernel on $K \rightarrow \infty, \epsilon, A_{n}$, and $I_{n}$ is denoted explicitly using the subscript.

Using this stochastic kernel, the evolution of the density just before the $n$th impulse to that just before the $(n+1)$ th impulse is determined by

$$
h_{n+1}(\phi)=\int_{0}^{1} g_{\infty, \epsilon, A_{n}, I_{n}}\left(\phi ; \phi_{n}\right) h_{n}\left(\phi_{n}\right) d \phi_{n}=\mathcal{P}_{\infty, \epsilon, A_{n}, I_{n}} h_{n}(\phi),
$$

where $h_{n}$ is the density of the phase equation just before the $n$th impulse. We call the $\mathcal{P}_{\infty, \epsilon, A, I}$ operator a 1D SPTO.

Using the stochastic kernel for the full equation [see Eq. (8) in [5]], the SPTO that expresses the relationship between the density just before the $n$th impulse and that just before the $(n+1)$ th impulse is written as

$$
\begin{aligned}
h_{n+1}(r, \phi) & =\int_{0}^{1} \int_{0}^{\infty} g_{K, \epsilon, A_{n}, I_{n}}\left(r, \phi ; r_{n}, \phi_{n}\right) h_{n}\left(r_{n}, \phi_{n}\right) d r_{n} d \phi_{n} \\
& =\mathcal{P}_{K, \epsilon, A_{n}, I_{n}} h_{n}(r, \phi),
\end{aligned}
$$

where $h_{n}$ is the density just before the $n$th impulse for the full equation. It should be noted that we use $h$ as the density for both the full and phase equations. When these densities need to be distinguished, we show the variables of $h$ explicitly. The
$\mathcal{P}_{K, \epsilon, A, I}$ operator is referred to as a $2 \mathrm{D}$ SPTO, which is a $2 \mathrm{D}$ generalization of the phase transition curve with a stochastic term. In what follows, we use $\mathcal{P}_{K, \epsilon, A, I}$ for $K \in(0, \infty]$.

\section{Spectral properties of the SPTO}

The SPTO is a linear operator and contains all the information about the density evolution. In what follows, we discretize the SPTO to analyze its properties. We use numerical integration to approximate the integral equations, Eqs. (15) and (16). In the case of Eq. (16), we first truncate the integration range of the $r$ axis, since the density in the direction of $r$ decreases rapidly to zero as $r$ increases. The integration range for $r_{n}$ is large enough to approximate the integral equation as

$h_{n+1}(r, \phi) \simeq \int_{0}^{1} \int_{0}^{a} g_{K, \epsilon, A_{n}, I_{n}}\left(r, \phi ; r_{n}, \phi_{n}\right) h_{n}\left(r_{n}, \phi_{n}\right) d r_{n} d \phi_{n}$.

We then discretize Eq. (17) using quadrature rules:

$$
\begin{aligned}
h_{n+1}\left(r_{k}, \phi_{l}\right) \simeq & \sum_{j=1}^{N_{\phi_{n}}} \sum_{i=1}^{N_{r_{n}}} w_{i}^{r_{n}} w_{j}^{\phi_{n}} g_{K, \epsilon, A_{n}, I_{n}}\left(r_{k}, \phi_{l} ; r_{n, i}, \phi_{n, j}\right) \\
& \times h_{n}\left(r_{n, i}, \phi_{n, j}\right) .
\end{aligned}
$$

Legendre-Gauss quadrature for the integration with respect to $r_{n}$ and the trapezoidal rule for the integration with respect to $\phi_{n}$ are used; the same nodes are used for the approximation, i.e., the pair $\left(r_{n}, \phi_{n}\right)$ and $(r, \phi)$ have the same nodes.

The numerical calculations were performed with MATLAB. To calculate the density evolution, we set the discretization of the density $h(r, \phi)$ as follows:

$$
\left(\begin{array}{ccc}
h\left(r_{1}, \phi_{1}\right) & \ldots & h\left(r_{1}, \phi_{N_{\phi_{n}}}\right) \\
\vdots & \ddots & \vdots \\
h\left(r_{N_{r_{n}}}, \phi_{1}\right) & \ldots & h\left(r_{N_{r_{n}}}, \phi_{N_{\phi_{n}}}\right)
\end{array}\right)
$$

We transform this matrix as

$$
\left(\begin{array}{c}
h\left(r_{1}, \phi_{1}\right) \\
\vdots \\
h\left(r_{N_{r_{n}}}, \phi_{1}\right) \\
\vdots \\
h\left(r_{1}, \phi_{N_{\phi_{n}}}\right) \\
\vdots \\
h\left(r_{N_{r_{n}}}, \phi_{N_{\phi_{n}}}\right)
\end{array}\right) .
$$

To calculate the density evolution using Eq. (20), we constructed the corresponding stochastic matrix given as

$$
\left(\begin{array}{ccccccc}
g_{K, \epsilon, A, I, 1,1,1,1} & \cdots & g_{K, \epsilon, A, I, 1,1, N_{r_{n}}, 1} & \cdots & g_{K, \epsilon, A, I, 1,1,1, N_{\phi_{n}}} & \cdots & g_{K, \epsilon, A, I, 1,1, N_{r_{n}}, N_{\phi_{n}}} \\
\vdots & \ddots & \vdots & \ddots & \vdots & \ddots & \vdots \\
g_{K, \epsilon, A, I, N_{r_{n}}, 1,1,1} & \cdots & g_{K, \epsilon, A, I, N_{r_{n}}, 1, N_{r_{n}}, 1} & \cdots & g_{K, \epsilon, A, I, N_{r_{n}}, 1,1, N_{\phi_{n}}} & \cdots & g_{K, \epsilon, A, I, N_{r_{n}}, 1, N_{r_{n}}, N_{\phi_{n}}} \\
\vdots & \ddots & \vdots & \ddots & \vdots & \ddots & \vdots \\
g_{K, \epsilon, A, I, 1, N_{\phi_{n}}, 1,1} & \cdots & g_{K, \epsilon, A, I, 1, N_{\phi_{n}}, N_{r_{n}}, 1} & \cdots & g_{K, \epsilon, A, I, 1, N_{\phi_{n}}, 1, N_{\phi_{n}}} & \cdots & g_{K, \epsilon, A, I, 1, N_{\phi_{n}}, N_{r_{n}}, N_{\phi_{n}}} \\
\vdots & \ddots & \vdots & \ddots & \vdots & \ddots & \vdots \\
g_{K, \epsilon, A, I, N_{r_{n}}, N_{\phi_{n}}, 1,1} & \cdots & g_{K, \epsilon, A, I, N_{r_{n}}, N_{\phi_{n}}, N_{r_{n}}, 1} & \cdots & g_{K, \epsilon, A, I, N_{r_{n}}, N_{\phi_{n}}, 1, N_{\phi_{n}}} & \cdots & g_{K, \epsilon, A, I, N_{r_{n}}, N_{\phi_{n}}, N_{r_{n}}, N_{\phi_{n}}}
\end{array}\right),
$$


where we set $g_{K, \epsilon, A, I, i, j, k, l}=g_{K, \epsilon, A, I}\left(v_{i}, \phi_{j} ; r_{k}, \phi_{l}\right) w_{k}^{r} w_{l}^{\phi}$ for a concise representation of the matrix. Usually, the stochastic matrix is defined as a square matrix in which each row consists of nonnegative real numbers that sum to 1 . However, for convenience, we set the stochastic matrix, which is a discretization of the SPTO, to a square matrix whose columns consist of non-negative real numbers and for which each column sums to 1 .

Similarly, we approximate Eq. (15) using the trapezoidal rule as

$$
h_{n+1}\left(\phi_{k}\right) \simeq \sum_{j=1}^{N_{\phi_{n}}} w_{j}^{\phi_{n}} g_{\infty, \epsilon, A_{n}, I_{n}}\left(\phi_{k} ; \phi_{n, j}\right) h_{n}\left(\phi_{n, j}\right) .
$$

In this case, the representations of the matrix and vectors are easily deduced from Eq. (22).

Let us fix the input parameters $A$ and $I$ of the SPTO. We analyze the spectral properties of the discretized SPTO because the dynamics of the SPTO matrix are determined by the eigenvalues and eigenfunctions (eigenvectors). Let $\left\{\alpha_{i}\right\}$ and $\left\{e_{i}\right\}$ be the eigenvalues of the discretized SPTO, sorted in descending order according to their moduli, and the corresponding eigenfunctions, respectively $\left[i=1,2, \ldots, N_{\phi_{n}}\right.$ or $N_{\phi_{n}} N_{r_{n}}$, where $N_{\phi_{n}}$ and $N_{\phi_{n}} N_{r_{n}}$ are the dimensions of the discretized SPTOs in Eqs. (15) and (16), respectively]. Since the stochastic kernel is positive and the discretized SPTO is a positive stochastic matrix, the properties of the matrix can be summarized as follows [34]:

I: $\alpha_{1}=1$ and has a multiplicity of 1 . The corresponding eigenfunction has a unique invariant density $h_{K, \epsilon, A, I}^{*}$ or is $e_{1}$ with positive coordinates, i.e., the discretized SPTO is ergodic.

II: $\left|\alpha_{i}\right|<1$ for all eigenvalues different from 1 .

Hence, the eigenvalues $\alpha_{i}$ and eigenfunctions $e_{i}$ with $i \geqq 2$ have transient dynamics or contain the "dynamic" information of the discretized SPTO, whereas the invariant density $h_{K, \epsilon, A, I}^{*}$ or $e_{1}$ has stationary dynamics or "static" information of the discretized SPTO. In other words, the invariant density shows the response of the oscillator to periodic impulses as time goes to infinity. Based on these properties, the discretized SPTO is decomposed into two parts:

$$
\mathcal{P}_{K, \epsilon, A, I}=\mathcal{V}_{K, \epsilon, A, I}+\mathcal{Q}_{K, \epsilon, A, I}
$$

where $\mathcal{V}_{K, \epsilon, A, I}$ represents the stationary dynamics, i.e., for a density $h, \mathcal{V}_{K, \epsilon, A, I} h=h_{K, \epsilon, A, I}^{*}$, and $\mathcal{Q}_{K, \epsilon, A, I}$ corresponds to the transient dynamics. It should be noted that the spectral decomposition in Eq. (23) also holds for the "original" SPTO, since the SPTO is a constrictive Markov operator. The constrictiveness means that $\mathcal{P}_{K, \epsilon, A, I}^{n} h$ does not concentrate on a set of very small or vanishing measures as $n \rightarrow \infty$ (see $[35,36]$ for an explanation of constrictiveness and Proposition 5.3.2 in [35] to verify the constrictiveness of the SPTO). In short, since the stochastic kernel of the SPTO is positive, the SPTO is asymptotically stable [35] and thus constrictive.

For Eq. (16), the discretization of $\mathcal{V}_{K, \epsilon, A, I}$ in Eq. (23) is expressed as follows:

$$
\mathbf{h}_{K, \epsilon, A, I}^{*} \mathbf{1}^{T},
$$

where $T$ denotes the transpose, 1 represents an $\left(N_{r_{n}} \times N_{\phi_{n}}\right)$ vector of 1 's, and $\mathbf{h}_{K, \epsilon, A, I}^{*}$ is

$$
\left(\begin{array}{c}
h_{K, \epsilon, A, I}^{*}\left(r_{1}, \phi_{1}\right) \\
\vdots \\
h_{K, \epsilon, A, I}^{*}\left(r_{N_{r_{n}}}, \phi_{1}\right) \\
\vdots \\
h_{K, \epsilon, A, I}^{*}\left(r_{1}, \phi_{N_{\phi_{n}}}\right) \\
\vdots \\
h_{K, \epsilon, A, I}^{*}\left(r_{N_{r_{n}}}, \phi_{N_{\phi_{n}}}\right)
\end{array}\right) .
$$

\section{Stochastic phase locking}

Let us set the eigenvalues of the discretized SPTO $\alpha_{i}=$ $\rho_{i} \exp \left(2 \pi j \kappa_{i}\right)$, with $j$ as the imaginary unit and where $\rho_{i}$ and $\kappa_{i}$ are the modulus and angle of $\alpha_{i}$, respectively, and the corresponding eigenfunctions $e_{i}$ to fixed values. Applying the discretized $\mathcal{P}_{K, \epsilon, A, I}$ to $e_{i}$ for a total of $k$ times yields

$$
\mathcal{P}_{K, \epsilon, A, I}^{k} e_{i}=\alpha_{i}^{k} e_{i}=\rho_{i}^{k} \exp \left(2 \pi j k \kappa_{i}\right) e_{i} \quad(k=1,2, \ldots)
$$

Based on the dynamic information of the discretized $\mathcal{P}_{K, \epsilon, A, I}$, that is, eigenvalues $\alpha_{i}$ and eigenfunctions $e_{i}$ with $i \geqslant 2$, Doi et al. [37] defined stochastic bifurcation as the abrupt (not smooth) change of the eigenvalues of the operator from complex to real values at a possible stochastic bifurcation point. Furthermore, they also defined stochastic phase locking as the response that satisfies the following condition in addition to the stochastic bifurcation condition: In a certain range, there exists an $i$ that satisfies

$$
\mathcal{P}_{K, \epsilon, A, I}^{p} e_{i}=\alpha_{i}^{p} e_{i}=\rho_{i}^{p} \exp \left(2 \pi j p \kappa_{i}\right) e_{i}=\rho_{i}^{p} e_{i} \quad(i \geqslant 2) .
$$

In this study, we use the second eigenvalue of the operator to define the stochastic bifurcation and stochastic phase locking for categorizing the dynamics of the stochastic Poincaré oscillator. The definition of the stochastic bifurcation is still in an active debate. For the detailed discussion of the stochastic bifurcation, please see [37-43].

\section{E. Contribution to the current state from the past states}

Let us consider a sequence of $n$ impulses and the corresponding product of discretized SPTOs $\mathcal{H}_{n, 1}=$ $\mathcal{P}_{K, \epsilon, A_{n}, I_{n}} \mathcal{P}_{K, \epsilon, A_{n-1}, I_{n-1}} \ldots \mathcal{P}_{K, \epsilon, A_{1}, I_{1}}$ that describes the response of the stochastic Poincaré oscillator to the impulses. We set the current state to the state just before the $(n+$ $1)$ th impulse, which is represented by $h_{n+1}\left(=\mathcal{H}_{n, 1} h_{1}\right)$. Since the current density is determined by the product of the discretized SPTOs, the current density depends on the past activity of the stochastic Poincaré oscillator. The structure of the products $\mathcal{P}_{K, \epsilon, A_{n}, I_{n}} \mathcal{P}_{K, \epsilon, A_{n-1}, I_{n-1}} \ldots \mathcal{P}_{K, \epsilon, A_{1}, I_{1}}$ tells us how the past activity affects the current density. 
Using Eq. (23), the product is expressed as follows [5]:

$$
\begin{aligned}
h_{n+1}= & \mathcal{H}_{n, 1} h_{1} \\
= & \left\{\mathcal{V}_{K, \epsilon, A_{n}, I_{n}}+\sum_{i=1}^{n-1}\left(\prod_{j=0}^{n-i-1} \mathcal{Q}_{K, \epsilon, A_{n-j}, I_{n-j}}\right) \mathcal{V}_{K, \epsilon, A_{i}, I_{i}}\right. \\
& \left.+\prod_{l=0}^{n-1} \mathcal{Q}_{K, \epsilon, A_{n-l}, I_{n-l}}\right\} h_{1} \\
= & h_{K, \epsilon, A_{n}, I_{n}}^{*}+\sum_{i=1}^{n-1}\left(\prod_{j=0}^{n-i-1} \mathcal{Q}_{K, \epsilon, A_{n-j}, I_{n-j}}\right) h_{K, \epsilon, A_{i}, I_{i}}^{*} \\
& +\prod_{l=0}^{n-1} \mathcal{Q}_{K, \epsilon, A_{n-l}, I_{n-l}} h_{1},
\end{aligned}
$$

where $h_{K, \epsilon, A_{i}, I_{i}}^{*}$ is the invariant density of $\mathcal{P}_{K, \epsilon, A_{i}, I_{i}}$, $(1 \leqslant$ $i \leqslant n)$. It should be noted that Eq. (26) holds for both "original" and "discretized" SPTOs since Eq. (23) holds for both. Equation (26) suggests that the invariant density at the last impulse, e.g., the invariant density of $\mathcal{P}_{K, \epsilon, A_{n}, I_{n}}$, always appears in the equation of the product as is. If all the transient components of each discretized SPTO in the product are zero matrices, then the density is always equal to the invariant density at the last impulse. The second term in Eq. (26) shows the effect of the difference between adjacent invariant densities, as the following equation holds for each term in the second term:

$$
\begin{aligned}
\mathcal{Q}_{K, \epsilon, A_{i}, I_{i}} \mathcal{V}_{K, \epsilon, A_{i-1}, I_{i-1}} h_{1} & =\mathcal{Q}_{K, \epsilon, A_{i}, I_{i}} h_{K, \epsilon, A_{i-1}, I_{i-1}}^{*} \\
& =\mathcal{P}_{K, \epsilon, A_{i}, I_{i}} h_{K, \epsilon, A_{i-1}, I_{i-1}}^{*}-h_{K, \epsilon, A_{i}, I_{i}}^{*} .
\end{aligned}
$$

This means that the contribution of this term becomes small if the difference between adjacent invariant densities is small. The third term in Eq. (26) describes the dependence on the initial density.

Figure 1 illustrates how each term in the second and third terms of Eq. (26) affect the current density. For example, $\mathcal{Q}_{K, \epsilon, A_{n}, I_{n}} \mathcal{V}_{K, \epsilon, A_{n-1}, I_{n-1}}$ is produced by $\mathcal{P}_{K, \epsilon, A_{n}, I_{n}} \mathcal{P}_{K, \epsilon, A_{n-1}, I_{n-1}}$, since $\mathcal{V}_{K, \epsilon, A_{i}, I_{i}}$ and $\mathcal{Q}_{K, \epsilon, A_{i}, I_{i}}$ are from $\mathcal{P}_{K, \epsilon, A_{i}, I_{i}}(1 \leqslant i \leqslant n)$, respectively. In other words, $\mathcal{Q}_{K, \epsilon, A_{n}, I_{n}} \mathcal{V}_{K, \epsilon, A_{n}, I_{n}}$ is determined by the input parameters of the $n$th and $(n-1)$ th impulses. Thus, we treat the term $\mathcal{Q}_{K, \epsilon, A_{n}, I_{n}} \mathcal{V}_{K, \epsilon, A_{n-1}, I_{n-1}}$ as the effect produced by the $(n-1)$ th and $n$th impulses as in Fig. 1 . In this way, we can attribute the past neuronal activity of the stochastic Poincaré oscillator to the components in Eq. (26). To evaluate the relative contribution of each term that contains information about past activity, we use the following one-norm of a discretized operator $B=\left(b_{i j}\right)$ :

$$
\|B\|_{1}=\sup _{\mathbf{x} \neq \mathbf{0}} \frac{\|B \mathbf{x}\|_{1}}{\|\mathbf{x}\|_{1}}=\max _{1 \leqslant j \leqslant m} \sum_{i=1}^{m}\left|b_{i j}\right| w_{i},
$$

where $\mathbf{x}$ is a vector, and $\|\mathbf{x}\|_{1}=\sum_{i=1}^{m}\left|x_{i}\right| w_{i}$ ( $m$ is the dimension of $\mathbf{x}$ and $w_{i}$ is determined by Eq. (22) for $K=\infty$ and by Eqs. (18)-(21) for finite values of $K$, since the trapezoidal rule and quadrature are used for the numerical integration). Since the discretized SPTO is a positive matrix, the product of discretized SPTOs is weakly ergodic $[44,45]$.

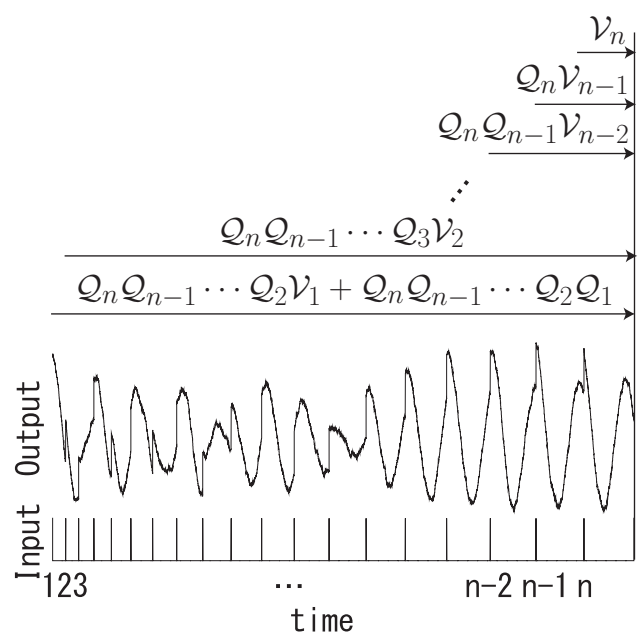

FIG. 1. Schematic diagram explaining the dependence of the current density on the past activity of the stochastic Poincare oscillator. The terms of the product of operators on the right-hand side of the second equation in Eq. (26) are plotted as a function of the input impulse number. The dynamics of the stochastic Poincaré oscillator are governed by the product of the stochastic phase transition operators (SPTOs) [for a detailed explanation of the SPTO, see Eqs. (15) and (16)], and the SPTO $\mathcal{P}_{K, \epsilon, A_{i}, I_{i}}$ expresses the relationship between the density just before the $i$ th impulse to that just before the $(i+1)$ th impulse: $\mathcal{P}_{K, \epsilon, A_{i}, I_{i}}=\mathcal{V}_{K, \epsilon, A_{i}, I_{i}}+\mathcal{Q}_{K, \epsilon, A_{i}, I_{i}}$, where $\mathcal{V}_{K, \epsilon, A_{i}, I_{i}}$ denotes the stationary dynamics and $\mathcal{Q}_{K, \epsilon, A_{i}, I_{i}}$ represents the transient dynamics. In this figure, the operators $\mathcal{V}_{i}$ and $\mathcal{Q}_{i}$ denote $\mathcal{V}_{K, \epsilon, A_{i}, I_{i}}$ and $\mathcal{Q}_{K, \epsilon, A_{i}, I_{i}}$, respectively. If all the transient components of each $\mathcal{P}_{K, \epsilon, A_{i}, I_{i}}$ in the product are zero operators, then the current density is determined by the invariant component of the last impulse $\mathcal{V}_{n}$. The "output" is the membrane potential of the stochastic Poincaré oscillator, and the "input" shows the input impulses added to the stochastic Poincaré oscillator. Abscissa is time.

The weak ergodicity leads to the following property for any densities $h$ and $h^{\prime}$ :

$$
\left\|\mathcal{H}_{n, n_{0}} h-\mathcal{H}_{n, n_{0}} h^{\prime}\right\|_{1} \rightarrow 0 \text { for all } n_{0} \text {, as } n \rightarrow \infty,
$$

where $\mathcal{H}_{n, n_{0}}=\mathcal{P}_{K, \epsilon, A_{n}, I_{n}} \mathcal{P}_{K, \epsilon, A_{n-1}, I_{n-1}} \ldots \mathcal{P}_{K, \epsilon, A_{n_{0}}, I_{n_{0}}}$, and $n_{0}$ and $n$ are positive integers with $n \geqslant n_{0}$. This means that the product of discretized SPTOs loses its dependence on the initial density. Because the third term in Eq. (26) is the only term that depends on the initial density, the one-norm of this term goes to zero as $n \rightarrow \infty$.

\section{F. Stochastic rotation number}

To connect the density evolution and the firing rate of the stochastic Poincaré oscillator, we calculate a stochastic rotation number for the phase equation in Eq. (8) and the full equation in Eq. (7) following the definition in $[23,46]$. Considering the case for which the $n$th impulse is added at $\phi_{n}$, the lifted angular coordinate from Eq. (13) just before the $(n+1)$ th impulse is distributed as follows:

$$
n\left[\theta ; \tilde{\Theta}_{I_{n}}^{(0)}\left(\phi_{n}\right), \epsilon^{2} \Sigma_{\tilde{\Theta} I_{n}}\left(\phi_{n}\right)\right],
$$

where it should be noted that $\theta \in \mathbb{R}$. That is, $\theta$ includes multiple rotations around the origin. The mean difference in the lifted angular coordinates of two consecutive impulses 
becomes

$$
\begin{aligned}
w_{\infty, A_{n}, I_{n}}\left(\phi_{n}\right) & =\int_{-\infty}^{\infty}\left(\theta-\phi_{n}\right) n\left[\theta ; \tilde{\Theta}_{I_{n}}^{(0)}\left(\phi_{n}\right), \epsilon^{2} \Sigma_{\tilde{\Phi} I_{n}}\left(\phi_{n}\right)\right] d \theta \\
& =\tilde{F}_{\Phi}\left(\phi_{n}\right)-\phi_{n}+I_{n}
\end{aligned}
$$

where the subscripts show the dependence on the parameters, the subscript $\infty$ denotes $K \rightarrow \infty$, and the mean angular coordinate difference depends on $A_{n}$ via $\tilde{F}_{\Phi}\left(\phi_{n}\right)$. We define an "instantaneous" stochastic rotation number in the interval just before the $n$th to just before the $(n+1)$ th impulses for the phase equation as follows:

$$
\begin{aligned}
\Omega_{\infty, \epsilon, A_{n}, I_{n}} & =\frac{1}{I_{n}} \int_{0}^{1} w_{\infty, A_{n}, I_{n}}\left(\phi_{n}\right) h_{n}\left(\phi_{n}\right) d \phi_{n} \\
& =1+\frac{1}{I_{n}} \int_{0}^{1}\left(\tilde{F}_{\Phi}\left(\phi_{n}\right)-\phi_{n}\right) h_{n}\left(\phi_{n}\right) d \phi_{n},
\end{aligned}
$$

where the subscripts of $\Omega_{\infty, \epsilon, A_{n}, I_{n}}$ represent the dependence of the instantaneous stochastic rotation number on the parameters $K \rightarrow \infty, \epsilon, A_{n}$, and $I_{n}$, respectively. The instantaneous stochastic rotation number depends on $\epsilon$ via $h_{n}\left(\phi_{n}\right)$. Note that $1: 1$ correspondence between the set of input parameters $A_{n}$, $I_{n}$, and $\Omega_{\infty, \epsilon, A_{n}, I_{n}}$ is achieved when $\epsilon$ is fixed. If the impulse amplitude and interimpulse interval do not vary with time, then the "steady-state" stochastic rotation number is

$$
\Omega_{\infty, \epsilon, A, I}=1+\frac{1}{I} \int_{0}^{1}\left(\tilde{F}_{\Phi}(\phi)-\phi\right) h_{\infty, \epsilon, A, I}^{*}(\phi) d \phi,
$$

where the subscripts $\infty, \epsilon, A$, and $I$ define $K \rightarrow \infty$, the noise strength, a constant amplitude, and a constant interimpulse interval, respectively; $h_{\infty, \epsilon, A, I}^{*}$ is the invariant density of $\mathcal{P}_{\infty, \epsilon, A, I}$.

Similarly, we define these stochastic rotation numbers for the full equation. In this case, we have to include the difference caused by the radial component of the density because the state points can move across the entire phase plane. According to Eq. (8) in [5], the state point just before the $(n+1)$ th impulse is distributed as

$$
\begin{aligned}
& n\left[(r, \theta) ; \mathbf{U}_{I_{n}}^{(0)}\left(r_{n}, \phi_{n}\right), \epsilon^{2} \Sigma_{I_{n}}\left(r_{n}, \phi_{n}\right)\right] \\
& \quad+n\left[(-r, \theta+0.5) ; \mathbf{U}_{I_{n}}^{(0)}\left(r_{n}, \phi_{n}\right), \epsilon^{2} \Sigma_{I_{n}}\left(r_{n}, \phi_{n}\right)\right],
\end{aligned}
$$

where $\mathbf{U}_{I_{n}}^{(0)}\left(r_{n}, \phi_{n}\right)=\left(R_{I_{n}}^{(0)}\left(r_{n}, \phi_{n}\right), \Theta_{I_{n}}^{(0)}\left(r_{n}, \phi_{n}\right)\right)$ shows the explicit dependence on $\left(r_{n}, \phi_{n}\right)$ (the state point just before the $n$th impulse), $r \in\{x ; x>0, x \in \mathbb{R}\}$, and $\theta \in \mathbb{R}$. Please note that the lifted angular coordinate is used in Eq. (34). The mean difference in the lifted angular coordinates of the $n$th and $(n+$ 1)th impulses is

$$
\begin{aligned}
w_{K, \epsilon, A_{n}, I_{n}}\left(r_{n}, \phi_{n}\right)= & \int_{0}^{\infty} \int_{-\infty}^{\infty}\left(\theta-\phi_{n}\right) n\left[(r, \theta) ; \mathbf{U}_{I_{n}}^{(0)}\left(r_{n}, \phi_{n}\right), \epsilon^{2} \Sigma_{I_{n}}\left(r_{n}, \phi_{n}\right)\right] d \theta d r \\
& +\int_{0}^{\infty} \int_{-\infty}^{\infty}\left(\theta-\phi_{n}\right) n\left[(-r, \theta+0.5) ; \mathbf{U}_{I_{n}}^{(0)}\left(r_{n}, \phi_{n}\right), \epsilon^{2} \Sigma_{I_{n}}\left(r_{n}, \phi_{n}\right)\right] d \theta d r \\
= & {\left[F_{\Phi}\left(r_{n}, \phi_{n}\right)-\phi_{n}+I_{n}\right] \int_{0}^{\infty} n\left[r ; R_{I_{n}}^{(0)}\left(r_{n}, \phi_{n}\right), \epsilon^{2} E\left[A_{1 R I_{n}}^{2}\right]\right] d r } \\
& +\left[F_{\Phi}\left(r_{n}, \phi_{n}\right)-\left(\phi_{n}+0.5\right)+I_{n}\right] \int_{0}^{\infty} n\left[-r ; R_{I_{n}}^{(0)}\left(r_{n}, \phi_{n}\right), \epsilon^{2} E\left[A_{1 R I_{n}}^{2}\right]\right] d r,
\end{aligned}
$$

where the subscript of $w$ shows the dependence on the parameters $K, \epsilon$, the $n$th impulse amplitude $A_{n}$, and the $n$th impulse interimpulse interval $I_{n}$. The stochastic rotation number between the $n$th and $(n+1)$ th impulses is

$$
\Omega_{K, \epsilon, A_{n}, I_{n}}=\frac{1}{I_{n}} \int_{0}^{1} \int_{0}^{\infty} w_{K, \epsilon, A_{n}, I_{n}}\left(r_{n}, \phi_{n}\right) h_{n}\left(r_{n}, \phi_{n}\right) d r_{n} d \phi_{n} .
$$

This value is an instantaneous stochastic rotation number for the full equation. We can also define the steady-state stochastic rotation number for the full equation as follows:

$$
\Omega_{K, \epsilon, A, I}=\frac{1}{I} \int_{0}^{1} \int_{0}^{\infty} w_{K, \epsilon, A, I}(r, \phi) h_{K, \epsilon, A, I}^{*}(r, \phi) d r d \phi,
$$

where $h_{K, \epsilon, A, I}^{*}$ is the invariant density of $\mathcal{P}_{K, \epsilon, A, I}$. Since $\Omega_{K, \epsilon, A_{n}, I_{n}}$ is the stochastic rotation number between the $n$th and $(n+1)$ th impulses, it corresponds to an impulse amplitude $A_{n}$ and input interval $I_{n}$. Using this definition of the stochastic rotation number, an instantaneous input rate corresponds to a specific output rate. In this way, one can construct the instantaneous firing rate of the Poincaré oscillator as a function of the instantaneous input rate.

Furthermore, using the decomposition of $h_{n}\left(r_{n}, \phi_{n}\right)$ by Eq. (26), Eq. (36) can be written as

$$
\begin{aligned}
\Omega_{K, \epsilon, A_{n}, I_{n}}= & \int_{0}^{1} \int_{0}^{\infty} w_{K, \epsilon, A_{n}, I_{n}}\left(r_{n}, \phi_{n}\right) h_{K, \epsilon, A_{n-1}, I_{n-1}}^{*}\left(r_{n}, \phi_{n}\right) d r_{n} d \phi_{n} \\
& +\int_{0}^{1} \int_{0}^{\infty} w_{K, \epsilon, A_{n}, I_{n}}\left(r_{n}, \phi_{n}\right) \sum_{i=0}^{n-2}\left(\prod_{j=0}^{n-i-2} \mathcal{Q}_{K, \epsilon, A_{n-1-j}, I_{n-1-j}}\right) h_{K, \epsilon, A_{i}, I_{i}}^{*}\left(r_{n}, \phi_{n}\right) d r_{n} d \phi_{n} \\
& +\int_{0}^{1} \int_{0}^{\infty} w_{K, \epsilon, A_{n}, I_{n}}\left(r_{n}, \phi_{n}\right) \prod_{l=0}^{n-2} \mathcal{Q}_{K, \epsilon, A_{n-1-l}, I_{n-1-l}} h_{1}\left(r_{n}, \phi_{n}\right) d r_{n} d \phi_{n},
\end{aligned}
$$


where the operator $\mathcal{Q}_{K, \epsilon, A, I}$ is from the original SPTO, i.e., not from the discretized SPTO. Equation (38) tells us that the stochastic rotation number is determined by three terms. The first term is a contribution by the invariant density at the $(n-1)$ th impulse. The second term is the contribution produced by the invariant densities corresponding to the input parameters $\left\{A_{1}, I_{1}\right\}, \ldots,\left\{A_{n-2}, I_{n-2}\right\}$ with a weight determined by the corresponding transient components of the SPTO. The third component is from the initial density. The contribution of the initial density is also weighted by the transient components of the SPTO corresponding to given input impulses. Similarly, the decomposition of Eq. (32) becomes

$$
\begin{aligned}
\Omega_{\infty, \epsilon, A_{n}, I_{n}}= & \int_{0}^{1} \int_{0}^{\infty} w_{\infty, A_{n}, I_{n}}\left(\phi_{n}\right) h_{\infty, \epsilon, A_{n-1}, I_{n-1}}^{*}\left(\phi_{n}\right) d r_{n} d \phi_{n} \\
& +\int_{0}^{1} \int_{0}^{\infty} w_{\infty, A_{n}, I_{n}}\left(\phi_{n}\right) \sum_{i=0}^{n-2}\left(\prod_{j=0}^{n-i-2} \mathcal{Q}_{\infty, \epsilon, A_{n-1-j}, I_{n-1-j}}\right) h_{\infty, \epsilon, A_{i}, I_{i}}^{*}\left(\phi_{n}\right) d \phi_{n} \\
& +\int_{0}^{1} \int_{0}^{\infty} w_{\infty, A_{n}, I_{n}}\left(\phi_{n}\right) \prod_{l=0}^{n-2} \mathcal{Q}_{\infty, \epsilon, A_{n-1-l}, I_{n-1-l}} h_{1}\left(\phi_{n}\right) d \phi_{n} .
\end{aligned}
$$

In what follows, we use $\Omega_{K, \epsilon, A, I}$ for $K \in(0, \infty]$, and we calculate $\Omega_{K, \epsilon, A, I}$ using the same numerical integration methods explained in the calculation of the spectral properties of the SPTO.

\section{G. Interspike interval density}

The ISI density is used to characterize the spiking activity of neurons in the steady state. Nesse et al. [46] calculated the ISI density of the phase model with multiplicative noise by considering a population of neuronal oscillators. We extend their idea to the case in which the neuronal dynamics is written in terms of the stochastic differential equations. Specifically, we derive the ISI density for the reduced model Eq. (10). According to [46], the ISI density is derived in two steps: (1) calculation of the relative spike density that gives the time of the next input impulse arrival after a spike of the reduced model, and (2) calculation of the conditional ISI density relative to the first input impulse time. To calculate the ISI density, we make the same two assumptions as Nesse et al. [46]. The first assumption is that an impulse does not produce the normalized angular coordinate shift across unity. This is satisfied by assuming that an impulse makes an instantaneous horizontal shift by an amount equal to the impulse amplitude. The second assumption is that the interimpulse interval is large enough such that the normalized angular coordinate of the next impulse is not behind that of the previous impulse.

We consider impulses with a constant amplitude and interimpulse interval. For the calculation of the relative spike density, we set the density just before the first input impulse to the invariant density $h_{K, \epsilon, A, I}^{*}$, where $A$ and $I$ are the constant input amplitude and interimpulse interval, respectively. We write the relative spike density as $p_{S p, I m}(\tau)$, where $\tau$ is the relative time from the spike of the reduced model $(\mathrm{Sp})$ to the next impulse $(\mathrm{Im})$. To calculate $p_{S p, I m}(\tau)$, we have to take into account the possibility that the phase model does not fire during some input impulses (refer to Fig. 1 in [46]). Using the derivation method outlined in [46], $p_{S p, I m}(\tau)$ for the phase equation is given as

$$
p_{S p, I m}(\tau)=\sum_{j=1}^{\infty} p_{S p, I m}^{j}(\tau), \tau \in[0, \min \{I, 1\}),
$$

where

$$
\mathcal{P}_{K, \epsilon, A, I, p} h(\phi)=\int_{0}^{1} n\left[\phi+p ; \tilde{\Theta}_{I}^{(0)}(\phi), \epsilon^{2} \Sigma_{\tilde{\Theta}_{I}}(\phi)\right] h(\phi) d \phi,
$$

$$
\begin{gathered}
\mathcal{P}_{K, \epsilon, A, I}=\sum_{p=-\infty}^{p=+\infty} \mathcal{P}_{K, \epsilon, A, I, p}, \\
p_{S p, I m}^{k}(\tau)=\mathcal{P}_{K, \epsilon, A, I, 1} \mathcal{P}_{K, \epsilon, A, I, 0}^{(k-1)} h_{K, \epsilon, A, I}^{*}(\tau),
\end{gathered}
$$

and $\mathcal{P}_{K, \epsilon, A, I, p} h(\phi)$ is the probability density of neurons that fire $p$ times between two input impulses with constant $A$ and $I$. The superscript $(k-1)$ in Eq. (43) indicates that $\mathcal{P}_{K, \epsilon, A, I, 0}$ is raised to the $(k-1)$ power, and $p_{S p, I m}^{j}(\tau)$ is the density of the relative time from the current spike to the next impulse after the preceding $j$ impulses.

The conditional ISI density relative to the first impulse time $\tau$ is denoted by $p_{S p, S p}(T \mid \tau)$, where $T$ is the time between successive output spikes of the reduced model relative to $\tau$. When the reduced model receives a single impulse between consecutive spikes, i.e., $T \in(\tau, I+\tau)$, we obtain the following relations (refer to Fig. 2 in [46]):

$$
\begin{gathered}
T=I+\tau-\psi, \\
1+\psi=\tilde{F}_{\Phi}(\tau)+I+\epsilon A_{1 \tilde{\Theta} I},
\end{gathered}
$$

where we have approximated Eq. (10) as

$$
\tilde{\Theta}_{I}^{(\epsilon)} \simeq \tilde{\Theta}_{I}^{(0)}+\epsilon A_{1 \tilde{\Theta} I}=\tilde{F}_{\Phi}(\tau)+I+\epsilon A_{1 \tilde{\Theta} I},
$$

to derive Eq. (45). Equation (44) is a result of the relationship between the ISI and impulses' normalized angular coordinate, while Eq. (45) comes from the relationship between the normalized angular coordinates $\psi$ and $\tau$. If the model is given by stochastic differential equations, then the approximation in Eq. (46) is necessary so that the density of $A_{1 \tilde{\Theta} I}$ is included in the following calculation. Using the probability density of $A_{1 \tilde{\Theta} I}$, i.e., the first term in Eq. (12), we obtain the conditional ISI density for $T \in(\tau, I+\tau)$ :

$$
p_{S p, S p}^{1}(T \mid \tau)=n\left[T ; 1+\tau-\tilde{F}_{\Phi}(\tau), \epsilon^{2} \Sigma_{\tilde{\Theta} I}(\tau)\right] .
$$


In a similar manner, if two impulses exist between consecutive spikes (i.e., $T \in[I+\tau, 2 I+\tau)$ ), then

$$
\begin{gathered}
T=2 I+\tau-\psi, \\
1+\psi=\tilde{F}_{\Phi}\left(\phi_{1}\right)+I+\epsilon A_{1 \tilde{\Theta} I}, \\
\phi_{1}=\tilde{F}_{\Phi}(\tau)+I+\epsilon A_{1 \tilde{\Theta} I} .
\end{gathered}
$$

The conditional density for this case is

$$
\begin{aligned}
p_{S p, S p}^{2}(T \mid \tau)= & \int_{I}^{\infty} n\left[T ; t^{\prime}+\tau-\left[\tilde{F}_{\Phi}\left(\phi_{1}\left(t^{\prime}+\tau\right)\right)\right.\right. \\
& \left.\left.-\phi_{1}\left(t^{\prime}+\tau\right)\right], \epsilon^{2} \Sigma_{\tilde{\Theta} I}\left(\phi_{1}\left(t^{\prime}+\tau\right)\right)\right] \\
& \times p_{S p, S p}^{1}\left(t^{\prime}+\tau \mid \tau\right) d t^{\prime}
\end{aligned}
$$

where $T \in[I+\tau, 2 I+\tau)$, and $\phi_{1}(t)=1-(t-\tau)+I$. In general, the conditional ISI density for $T \in[(j-1) I+$ $\tau, j I+\tau)$ is

$$
\begin{aligned}
p_{S p, S p}^{j}(T \mid \tau)= & \int_{(j-1) I}^{\infty} n\left[T ; t^{\prime}+\tau-\left[\tilde{F}_{\Phi}\left(\phi_{(j-1)}\left(t^{\prime}+\tau\right)\right)\right.\right. \\
& \left.\left.-\phi_{(j-1)}\left(t^{\prime}+\tau\right)\right], \epsilon^{2} \Sigma_{\tilde{\Theta} I}\left(\phi_{(j-1)}\left(t^{\prime}+\tau\right)\right)\right] \\
& \times p_{S p, S p}^{(j-1)}\left(t^{\prime}+\tau \mid \tau\right) d t^{\prime}
\end{aligned}
$$

where $\phi_{j}(t)=1-(t-\tau)+j I$

The conditional ISI density relative to the first impulse time $\tau$ is then given by

$$
p_{S p, S p}(T \mid \tau)=p_{S p, S p}^{j}(T \mid \tau), \quad T \in[(j-1) I+\tau, j I+\tau) .
$$

For the case in which there are two consecutive spikes of the reduced model, $\int_{0}^{1} p_{S p, I m}(T \mid \tau) d \tau$ reveals the fraction of reduced models that receive at least one impulse, while $1-$ $\int_{0}^{1} p_{S p, I m}(\tau) d \tau$ reveals the fraction that receive no impulse. Reduced models that receive no impulse generates spikes with a mean period of 1 . If the reduced model receives two impulses with zero amplitude at normalized angular coordinates of 0 and 1, then Eq. (46) means that the following relation should hold:

$$
1=I+\epsilon A_{1 \tilde{\Theta} I} .
$$

Since these two zero-amplitude impulses are added at the normalized angular coordinates of 0 and 1 , the interimpulse interval $I$ is equal to the ISI, $T=I$. Thus, the ISI density without impulses is

$$
n\left[T, 1, \epsilon^{2} \Sigma_{\tilde{\Theta} I}(0)\right] .
$$

Note that we have not made an approximation with the $\delta$ function as in [46], since the ISI density without impulses cannot be derived in this way. Thus, the ISI density $p_{S p, S p}(T)$ becomes

$$
\begin{aligned}
p_{S p, S p}(T)= & \int_{0}^{1} p_{S p, S p}(T \mid \tau) p_{S p, I m}(\tau) d \tau \\
& +n\left[T ; 1, \epsilon^{2} \Sigma_{\tilde{\Theta} I}(0)\right]\left(1-\int_{0}^{1} p_{S p, I m}(\tau) d \tau\right) .
\end{aligned}
$$

We used the trapezoidal rule to calculate Eq. (56) for the results presented here. For the full equation [Eq. (9)], the (a)

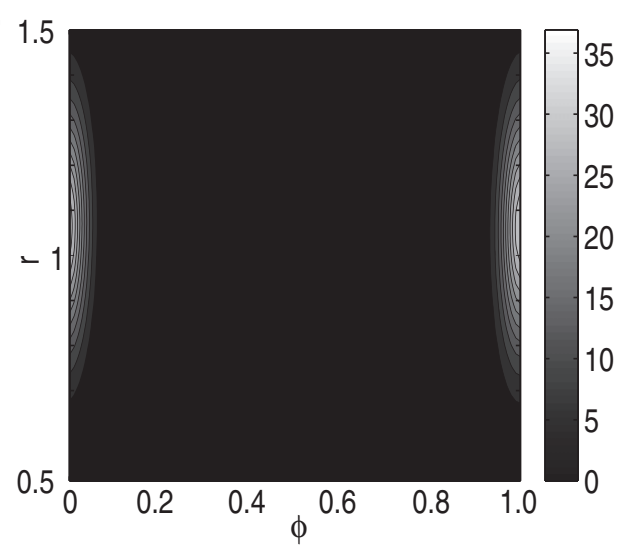

(b)
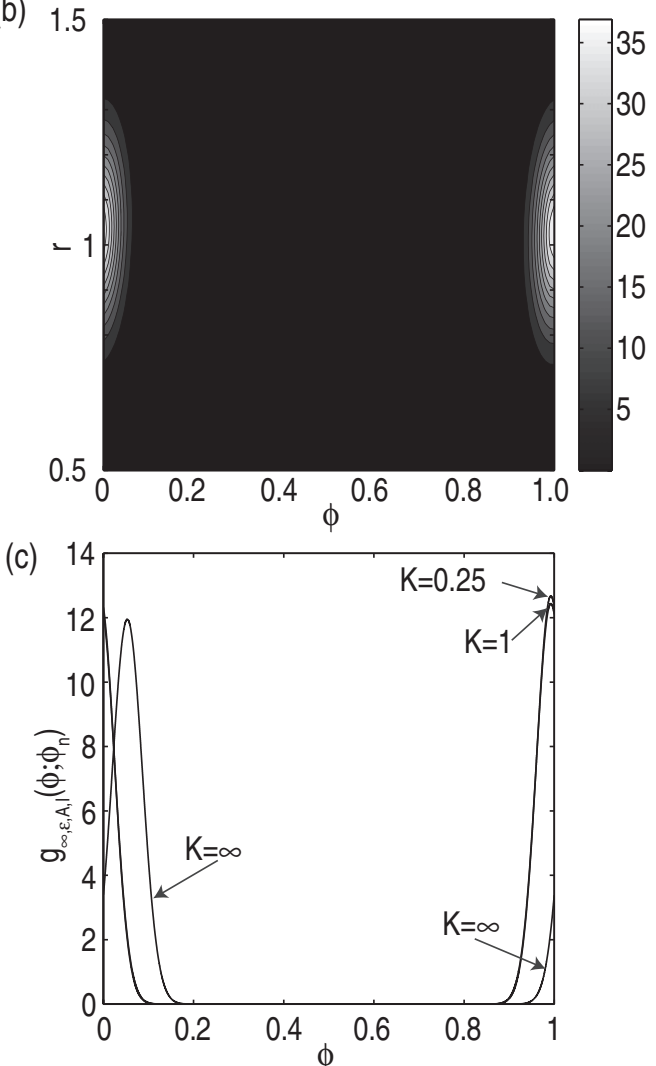

FIG. 2. Stochastic kernels. Stochastic kernels with (a) $K=0.25$ and (b) $K=1$. In (c), stochastic kernel with $K=\infty$ (indicated by two arrows with $K=\infty$ ) and marginal stochastic kernels of the normalized angular coordinate corresponded to stochastic kernels in (a) (indicated by an arrow with $K=0.25$ ) and (b) (indicated by an arrow with $K=1$ ), respectively. In (a) and (b), stochastic kernels were calculated using Eq. (8) in [5] and, in (c), stochastic kernel was calculated using Eq. (14). Marginal stochastic kernels of the normalized angular coordinate were calculated by numerically integrating Eq. (8) in [5] with respect to the radial coordinate. For the integration, Legendre-Gauss quadrature was used. The parameters for the stochastic kernels were $A=0.95, I=0.95, \epsilon=0.3$, with initial conditions of $\left(r_{1}, \phi_{1}\right)=(0.3,0.2)$ for (a), (b) and $\phi_{1}=0.2$ for (c). In (a) and (b), the abscissa and ordinate are the normalized angular coordinate and radial coordinate of the stochastic Poincaré oscillator, respectively. The color bar shows the probability density of the stochastic kernel. In (c), the abscissa is the normalized angular coordinate, and the ordinate is the probability density of the stochastic kernel or the marginal stochastic kernels. 
variance-covariance matrix that corresponds to $\Sigma_{\tilde{\Theta} I}(0)$ in Eq. (56) depends on the radial variable. Thus, the ISI density cannot be derived as in the case of the reduced model. We derive the ISI density for the full equation by solving the full equation using Euler-Maruyama method.

\section{H. Input impulses}

To examine the relation between the changing speed of input rate and the dynamics of the stochastic Poincare oscillator, we use impulses whose amplitudes are constant, and the instantaneous input rate changes according to

$$
\begin{gathered}
f_{n}=1 / I_{n}=f_{\text {start }}+\frac{\left(f_{\text {end }}-f_{\text {start }}\right)}{N}(n-1), \\
f_{\text {step }}=\left(f_{\text {end }}-f_{\text {start }}\right) / N,
\end{gathered}
$$

where $n=1, \ldots, N+1$. The input rate of the 1 st impulse and that of the $(N+1)$ th impulse are $f_{\text {start }}$ and $f_{\text {end }}$, respectively. Here, $N$ determines the number of interimpulse intervals, and $f_{\text {step }}$ denotes the step size of the input rate change. By changing $f_{\text {start }}, f_{\text {end }}$, and $N$, we can investigate the response of the stochastic Poincaré oscillator to time-varying impulses.

\section{RESULTS}

\section{A. Stochastic kernels}

Figure 2 shows the stochastic kernels for $K=0.25$ in (a), 1 in (b), and $\infty$ in (c) calculated from the same initial state (in the case of $K=\infty$, the same initial normalized angular coordinate as in the finite $K$ case is used). Comparing the stochastic kernels for the finite relaxation rates, $K=0.25$ and 1 , we see that the shape of the kernel with the larger relaxation rate has induced a sharper unimodal density. To see the effect of the radial coordinate dynamics, in Fig. 2(c), we showed the marginal stochastic kernels of the normalized angular coordinate corresponded to the stochastic kernels in Figs. 2(a) and 2(b), respectively. The peak position of the stochastic kernel for $K=\infty$ and the ones of the marginal stochastic kernels were different. The peak values of the marginal stochastic kernels near $\phi=1$ decreased as $K$ increased.
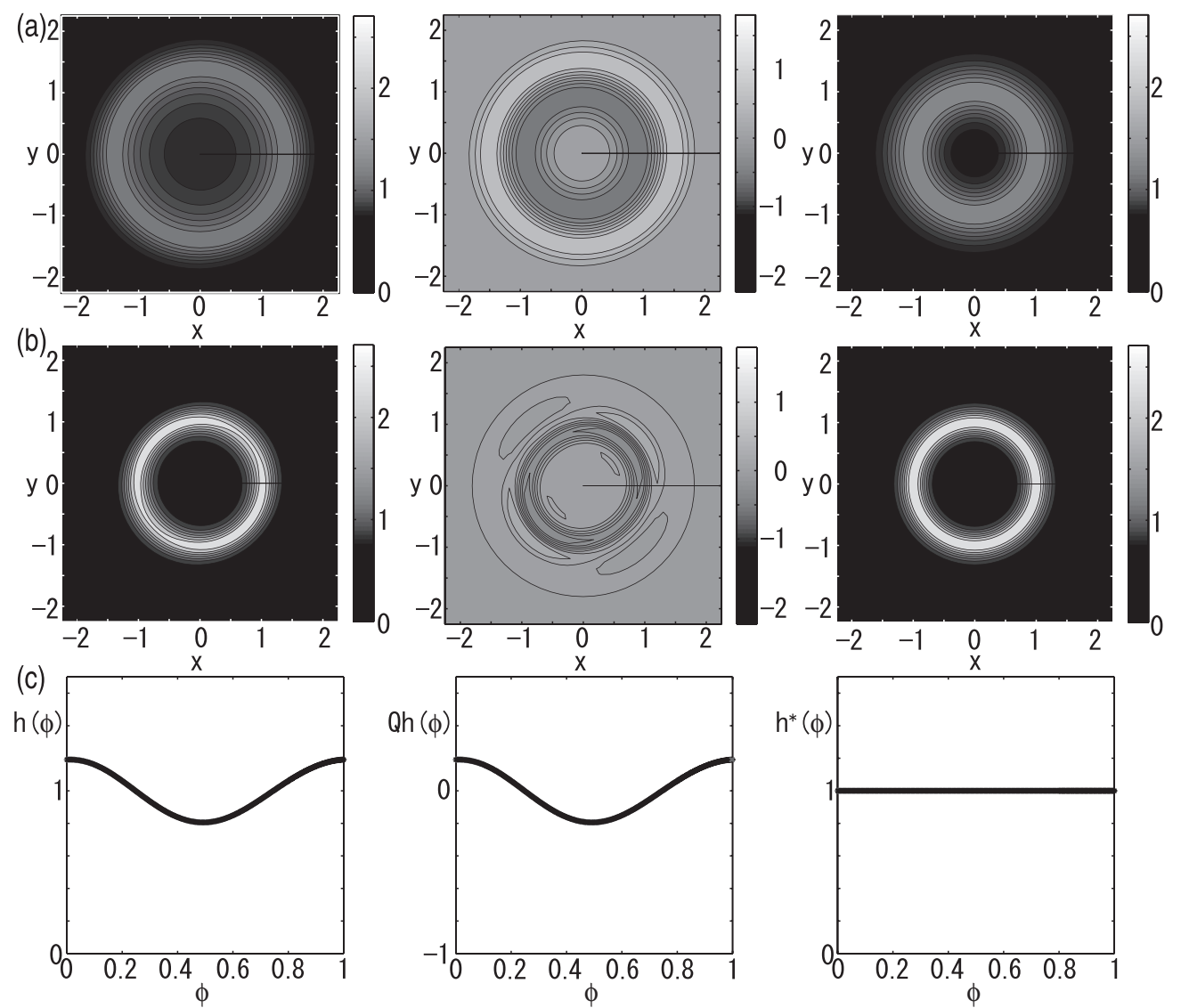

FIG. 3. Statistical global dynamics of the stochastic Poincaré oscillator without any impulse. The statistical phase plane dynamics of the stochastic Poincaré oscillator are shown by the probability density [left column; calculated using Eq. (18) for (a) and (b) and Eq. (22) for (c)], the corresponding transient components [middle column; Eq. (23)], and the corresponding invariant density [right column; Eq. (23)] without any impulses, i.e., $A=0$ after a time interval of $I=2.75$ for (a), (b), and (c). The relaxation rates were (a) $K=0.25$, (b) $K=1$, and (c) $K=\infty$. For (a) and (b), the initial density $h_{1}\left(r_{1}, \phi_{1}\right)$ was a uniform distribution with a support of $\left(r_{1}, \phi_{1}\right) \in(0,3.5] \times[0,1)$, and for $(\mathrm{c})$, the initial density $h_{1}\left(\phi_{1}\right)$ was a uniform distribution with a support of $\phi_{1} \in(0.2,0.8]$. In all cases, $\epsilon=0.3$. The color bar shows the probability density or the corresponding transient component. In (a) and (b), abscissae and ordinates are the membrane potential and refractoriness of the stochastic Poincaré oscillator, respectively. In (c), the abscissa is the normalized angular coordinate and the ordinate is probability density (left and right panels) or transient component (middle panel). 


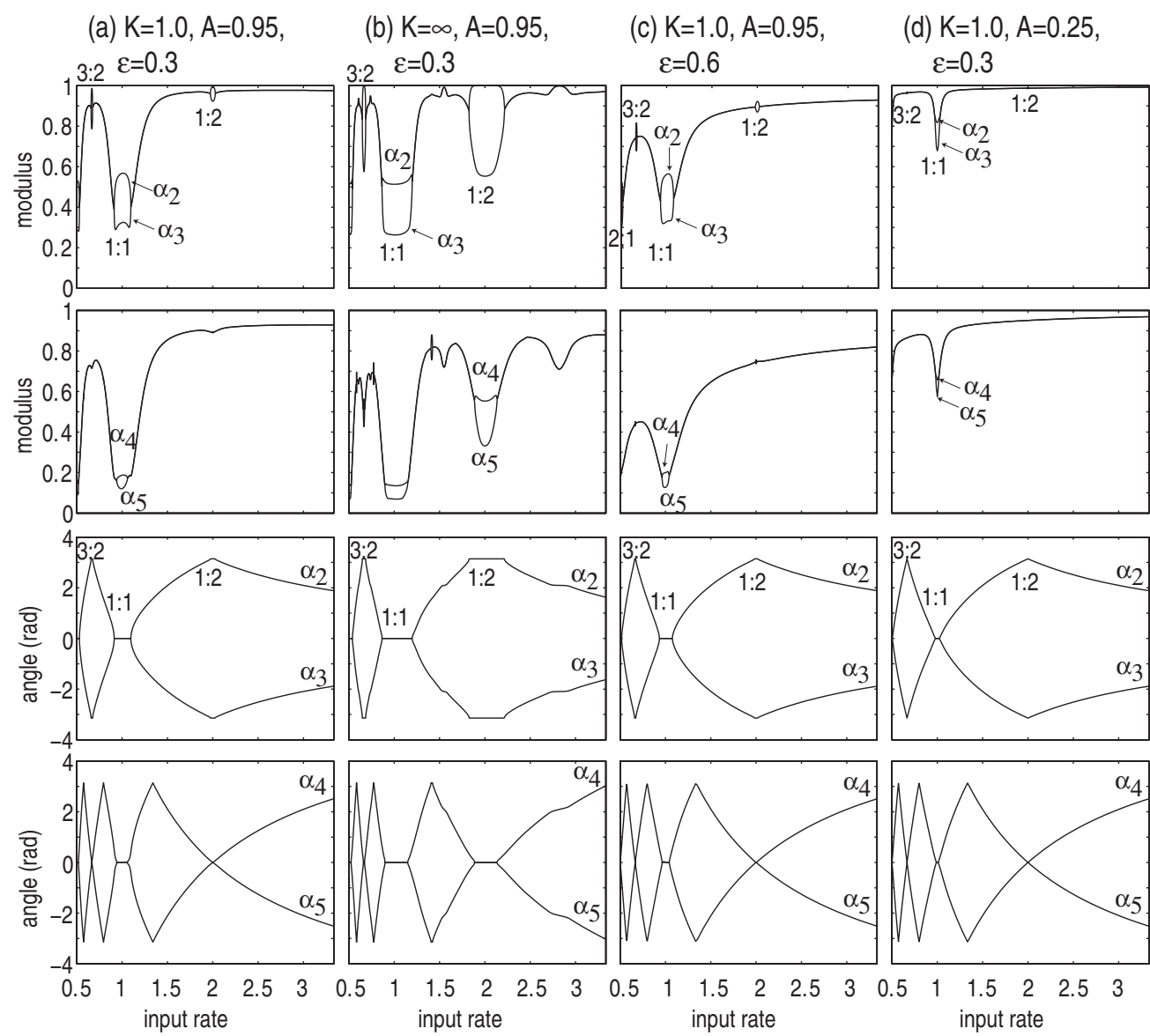

FIG. 4. Moduli and angles of the eigenvalues of the discretized SPTO as a function of the input rate. The eigenvalues were calculated using Eq. (18) for finite $K$ and Eq. (22) for infinite $K$. For each set of parameters, the moduli and angles of the second to fifth eigenvalues are plotted. Some stochastic phase-locking regions are labeled with their locking ratio. The parameters are shown in each panel. The input rate is on the abscissa and the moduli or angles of the eigenvalues of the discretized SPTO are on the ordinate.

\section{B. Density evolution}

If $A=0$, then the SPTO describes a density evolution that reflects the dynamics of the stochastic Poincaré oscillator itself. The spectral decomposition of the SPTO also decomposes the density into a transient component and an invariant density. Figure 3 shows the densities and corresponding transient components and invariant densities for different values of $K$. (The evolutions of the densities and transient components are shown in video S1 of the Supplemental Material [47].) Since the deterministic Poincaré oscillator has a stable limit cycle, the densities evolve toward this limit cycle and then converge to the corresponding invariant densities that are distributed around the limit cycle. The convergence speed depends on the relaxation rate of the stochastic Poincaré oscillator. That is, a larger relaxation rate is associated with a smaller transient component.

\section{Spectral properties of the SPTO}

If $K \rightarrow \infty$ and $\epsilon=0$, the Poincaré oscillator is known to exhibit typical structure in response to impulses with a constant $A$ and $I$. In particular, for $|A|<1$, the dynamics of the stochastic Poincare oscillator are described by the 1D phase transition curve of Eq. (4), which is an invertible diffeomorphism of the circle. For this case, the responses are classified into two categories: a phase locking, in which $q$ impulses correspond to $p$ spikes ( $p$ and $q$ are integer values), and a quasiperiodic response, where one impulse rotates the Poincaré oscillator an irrational number of times [20].

Using the definition of stochastic phase locking based on the eigenvalues of the discretized SPTO, we evaluated the effect of the relaxation rate, noise strength, impulse amplitude, and inverse of the interimpulse interval (input rate) on the response of the impulse-driven stochastic Poincaré oscillator. Figures 4(a) and 4(b) show the moduli and angles of the eigenvalues as a function of the input rate for different values of $K$. The overall trend of the moduli for both cases was to increase as the input rate increased, and some larger stochastic phase-locking regions survived even in the presence of noise. As $K$ increased, there was an overall decrease in the moduli of the eigenvalues, and the stochastic phase-locking regions became wider. In some stochastic phase-locking regions, the modulus of the second eigenvalue became larger as $K$ increased (for example, in the $1: 2$ and $3: 2$ stochastic phaselocking regions). Furthermore, in some regions, the modulus of the second eigenvalue took a value that was slightly smaller than 1 , as can be seen in the $1: 2$ stochastic phase-locking region. This indicates that the corresponding eigenfunction 
largely affects the dynamics in this region. In fact, the density tends to rotate in the phase plane, and the response can have a long transient regime even though this phenomenon depends on the initial density.

A comparison of Figs. 4(a) and 4(c) reveals how the moduli and angles of the eigenvalues of the discretized SPTO change in response to an increase in the noise strength, i.e., there was an overall decrease in the moduli of the eigenvalues but an increase in the modulus of the second eigenvalue around the $1: 1$ and $2: 1$ stochastic phase-locking regions. The stochastic phase-locking regions also became narrower. Thus, the detailed stochastic bifurcation structure disappears when the noise strength is increased.

Furthermore, the dependence of the moduli and angles of the eigenvalues of the discretized SPTO on the impulse amplitude can be seen in a comparison of Figs. 4(a) and 4(d). In this study, we concentrated on the impulse amplitude $|A|<1$, since an Arnold tongue structure exists in this range, at least for infinite $K$ [20], and this structure is a general structure of nonlinear oscillators. A comparison shows that the stochastic phase-locking region narrows when the impulse amplitude decreases. This is similar to the narrowing of the deterministic phase-locking region that is seen when the amplitude of the impulse decreases for $|A|<1$ and infinite $K$. An increase in the impulse amplitude resulted in an overall decrease in the moduli of the eigenvalues. However, the modulus of the second eigenvalue of the $1: 2$ and $3: 2$ stochastic phase-locking regions increased as an increase in the impulse amplitude.

To understand the type of stochastic bifurcation of the stochastic Poincaré oscillator with a finite relaxation rate, Fig. 5 shows the distribution of the first 15 eigenvalues of the discretized SPTO for finite $K(K=1)$. Some eigenvalues, whose moduli were not in the vicinity of zero and less than 1 , are located on the positive part of the $x$ axis throughout the stochastic bifurcation. We did not observe these eigenvalues in the case of the SPTO for $K=\infty$. These eigenvalues and the corresponding eigenfunctions describe the contribution of the radial transient component without oscillation by the application of the same SPTO. In relation to the definition of the stochastic bifurcation in terms of the distribution of the eigenvalues of the transition operator (in our case, this corresponds to the SPTO) [43], we also examined the eigenvalue distribution as a function of the input rate. We found that as the input rate decreased, the eigenvalue distribution exhibited "zipping" behavior, as shown in Fig. 5. That is, in a similar manner to closing a zip, the complex eigenvalues converge to real values as the input rate increases (left to right panel). It would seem that several spirals exist around the zero point but we were unable to determine the detailed structure around the zero point because the accuracy of the eigenvalues in this region was insufficient.

In the neighborhood of the stochastic $1: 1$ phase-locking region, the second and third eigenvalues were complex conjugates that correspond to stochastic quasiperiodic responses (these eigenvalues are indicated by the arrows in Fig. 5, left panel). For an input rate of 1.09630, the second eigenvalue was real, and for an input rate of 1.0, eigenvalues with moduli smaller than that of the second eigenvalue were real. Thus, a stochastic bifurcation occurs as the input rate decreases. We checked and confirmed that similar changes occur with a change in the input rate in the 1:2 stochastic phaselocking case. Thus, the Poincaré oscillator shows a stochastic saddle-node bifurcation, as defined in [37]. In addition, the shapes of the invariant densities did not change abruptly as a function of the input rate as has been reported for other systems [37-39,41,48] (the change in the invariant density as a function of the input rate is shown in video $\mathrm{S} 2$ of the Supplemental Material [47]).

\section{Stochastic rotation number}

The firing rate is an important statistic in spike-train analysis, and here, the counterparts of the firing rate are the instantaneous and steady-state stochastic rotation numbers. We used these numbers to understand the difference between the responses in the steady and transient states.

The curves in Fig. 6 show how the steady-state stochastic rotation number changes as a function of the input rate. In a $p: q$ stochastic phase-locking region, the slope of the stochastic rotation number was close to $p / q$. Since the invariant density changed smoothly as a function of the input rate, the steady-state stochastic rotation number did not show any abrupt changes at the edges of the stochastic phase-locking regions. As $K$ decreased, the stochastic phase-locking regions
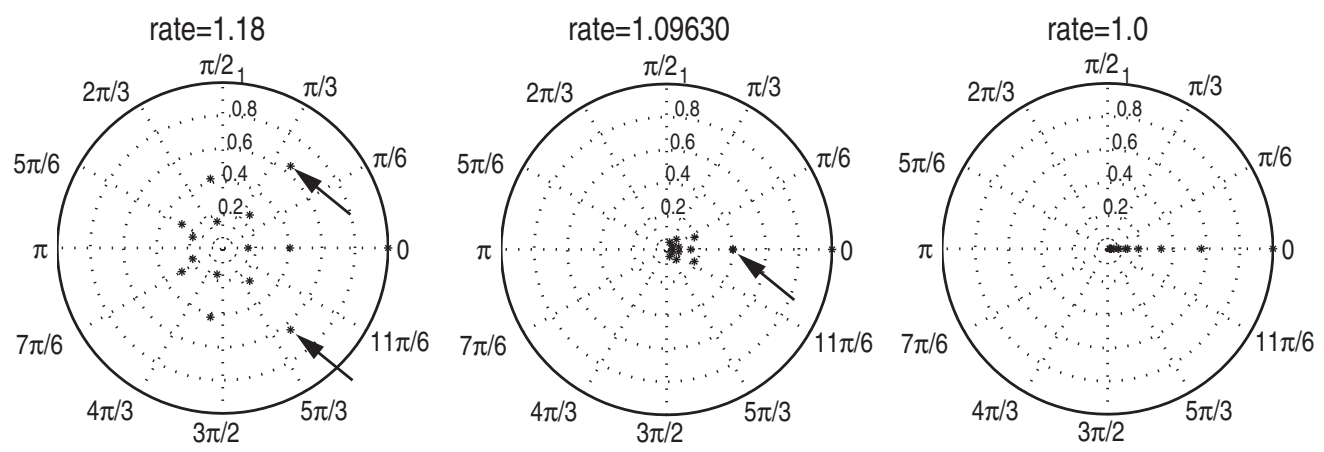

FIG. 5. Stochastic saddle-node bifurcation. The first 15 eigenvalues of the discretized SPTO [Eq. (18)] are plotted in the complex plane for the input rate indicated in each panel. The other parameters were $A=0.95, \epsilon=0.3$, and $K=1$. Angles of the eigenvalues of the discretized SPTO are in radians. In the left-hand panel, the arrows indicate the second and third eigenvalues. In the middle panel, the arrow indicates the second eigenvalue. As the input rate decreased, the second and third eigenvalues coincide, which is the stochastic saddle-node bifurcation. For more details about the stochastic bifurcation, see the text. 

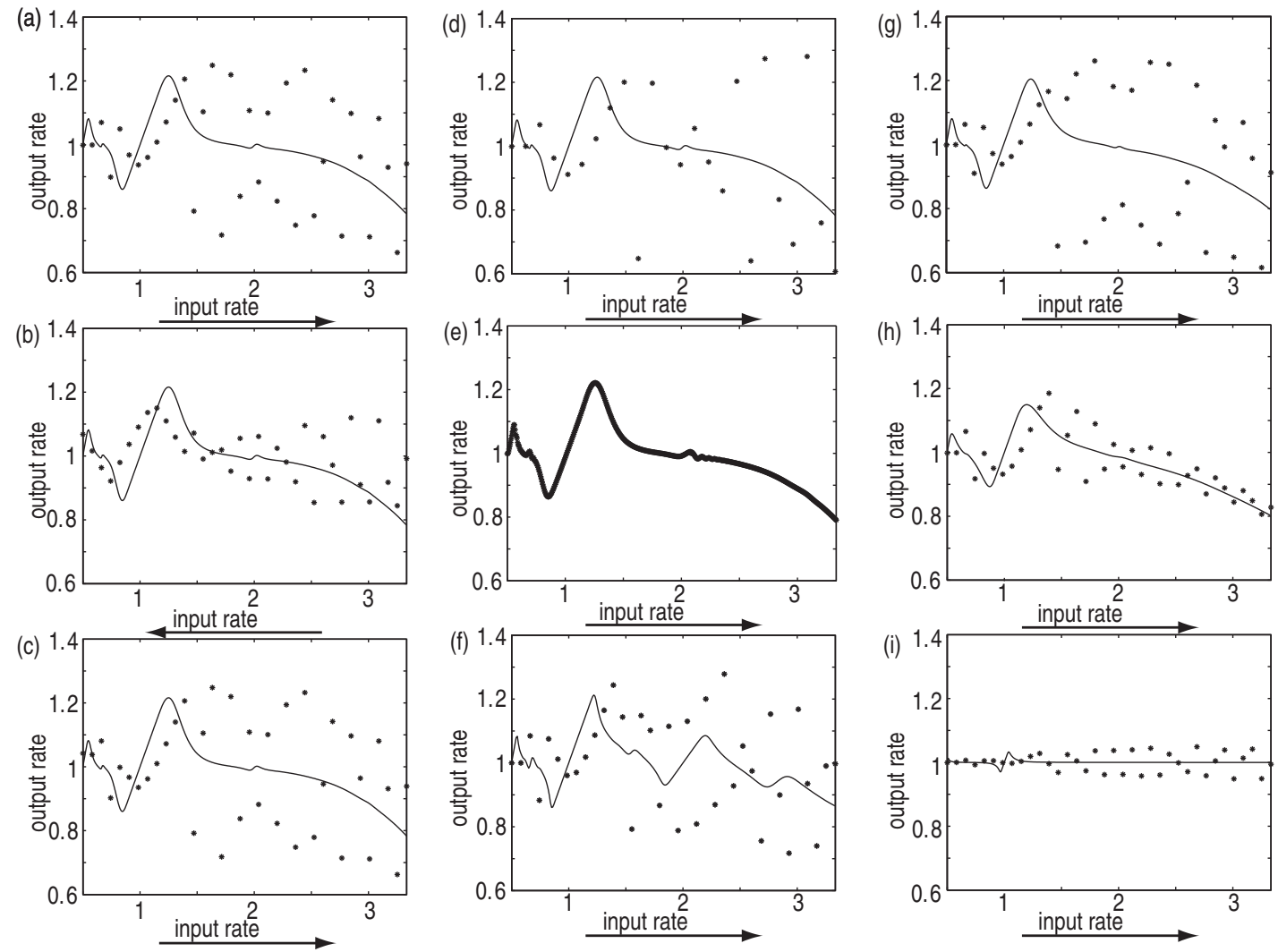

FIG. 6. Stochastic rotation number as a function of the input rate. The curves show the steady-state stochastic rotation number as a function of the input rate [calculated using Eq. (33) or (37)] and the asterisks show the instantaneous stochastic rotation number as a function of the input rate for different parameters [calculated using Eq. (32) or (36)]. We varied the relaxation rate, noise strength, and amplitude of the input impulse. (a) Standard input-output rate plot. The starting and final input rates were $f_{\text {start }}=0.5$ and $f_{\text {end }}=1 / 0.3,36$ impulses $(N=35)$ with an amplitude of $A=0.95$ were considered along with a relaxation rate of $K=1$ and noise strength of $\epsilon=0.3$. The initial density $h_{1}\left(r_{1}, \phi_{1}\right)$ was a uniform distribution with a support of $\left(r_{1}, \phi_{1}\right) \in(0,3.5] \times[0,1)$. In (b)-(i), the effects of various parameters on the stochastic rotation numbers are calculated by varying one parameter while the other parameters and initial density are the same as those in (a). Stochastic rotation numbers for (b) $f_{\text {start }}=1 / 0.3$ and $f_{\text {end }}=0.5$ to investigate the effect of $f_{\text {step }}$ in Eq. (58), (c) the initial density with a support of $\left(r_{1}, \phi_{1}\right) \in(0,3.5] \times[0.25,0.75)$ to examine the effect of the initial density, (d) $N=23$, (e) $N=500$, (f) $K=\infty,(\mathrm{g}) K=0.6$, (h) $\epsilon=0.6$, and (i) $A=0.25$. Arrow indicates the direction of the input rate in each panel.

narrower, and regions in which the slope was close to $p / q$ also narrowed [compare the curve in Fig. 6(a) for $K=1$ with that in Fig. 6(f) for $K=\infty$ ]. Furthermore, an increase in the noise strength and a decrease in the impulse amplitude in the $|A|<1$ range narrowed regions in which the slope was close to $p / q$ [compare Fig. 6(a) with Fig. 6(h) for the noise strength and Fig. 6(a) with Fig. 6(i) for the impulse amplitude].

If the time-varying impulses defined by Eq. (57) are added, then the response will have different properties from those of the steady state. We set $f_{\text {step }}$ to different values by changing $N$, $f_{\text {start }}$, and $f_{\text {end }}$ in Eq. (58) to investigate the dependence of the response on $f_{\text {step. }}$. Figures 6(a) and 6(b) show the instantaneous stochastic rotation number (asterisks) for different signs of $f_{\text {step }}$, but the absolute values of $f_{\text {step }}$, as well as the minimum and maximum input rates, were the same in both cases. Depending on whether the input rate increased or decreased, the stochastic rotation number showed different behavior. For example, if the input rate entered the $1: 1$ stochastic phaselocking region, then the instantaneous stochastic rotation number crossed the curve, that is, the steady-state stochastic rotation number, and took a smaller (larger) value than the steady-state stochastic rotation number as the input rate increased (decreased).

Figure 6(c) shows the instantaneous stochastic rotation numbers that originate from the different initial densities from Fig. 6(a) for the impulses whose input rates start from 0.5. After the first five impulses, the effect of the initial density was negligible.

If $\left|f_{\text {step }}\right|$ was small enough, then the instantaneous stochastic rotation number took a similar value to that of the steady state even though the modulus of the second eigenvalue was slightly smaller than 1 , as seen, for example, in the $1: 2$ stochastic phase-locking region [Fig. 6(e)]. Furthermore, since the product of the discretized SPTOs is weakly ergodic, the initial density is "forgotten" if the number of impulses is large enough, and this leads to the third term in Eq. (38) going to zero. Moreover, the invariant density changed smoothly as a function of the input rate, and thus the density $h_{K, \epsilon, A_{n-1}, I_{n-1}}^{*}$ was similar to $h_{K, \epsilon, A_{n}, I_{n}}^{*}$ in Eq. (38). Furthermore, according to Eq. (27), the individual terms in the second term of 
Eq. (38) become small if $\left|f_{\text {step }}\right|$ is small enough. This makes the instantaneous stochastic rotation number similar to the steady-state counterpart.

In the region where the modulus of the second eigenvalue was slightly less than 1 , the response produced by the discretized SPTO had a large transient component. This property induced a response that was different from the steady-state response even though the response depends on the initial density [Fig. 6, except for panel (e)]. For example, in Figs. 6(a)-6(c), the instantaneous stochastic rotation number oscillated and was largely different from the steady-state counterpart when the instantaneous input rate was roughly within $[1.5,1 / 0.3]$. The larger eigenvalues of the discretized SPTO in this range take mostly complex values, and their absolute values were close to 1 . This property leads to a difference in the instantaneous and steady-state stochastic rotation numbers and the oscillation of the instantaneous stochastic rotation number.

If we fix the range of the input rate and $f_{\text {step }}$ and examine results for a decrease in the impulse amplitude and an increase in the noise strength, we see that this leads to a smaller variation in the instantaneous stochastic rotation number [compare Fig. 6(a) with Figs. 6(i), and 6(h), respectively]. We also investigated the dependence of the instantaneous stochastic rotation number by changing the relaxation rate [Fig. 6(a) for $K=1$, Fig. 6(f) for $K=\infty$, and Fig. 6(g) for $K=0.6]$. As the relaxation rate increased beyond 0.4 , the variation of the instantaneous stochastic rotation number around the input rate 2 decreased for finite $K$ (we observed this tendency by checking $K \in[0.1,1.2]$ with step 0.1$)$. However, the variation around the input rate 2 increased as the relaxation rate increased if we compared Fig. 6(a) with Fig. 6(f).

\section{E. Interspike interval density}

To understand the response in the steady state, we calculated the ISI density. Figures 7(a)-7(c) show the ISI densities for $K=\infty$ and various input rates. In the $1: 1$ stochastic phaselocking region, the ISI density was unimodal, with the mean ISI similar to the input period. As the input rate decreased, the peak shifted to larger ISI values and decreased in magnitude; a plateau also appeared [Fig. 7(b)]. A further decrease in the input rate led to the appearance of three local maxima in the ISI density [Fig. 7(c)]. Figures 7(b) and 7(c) show the densities outside the $1: 1$ stochastic phase-locking region, and there is a stochastic bifurcation point between the densities shown in Figs. 7(a) and 7(b). However, the change in the ISI density was smooth because the ISI density is based on the invariant density of the corresponding SPTO. Figures 7(d)-7(f) show the ISI densities for $K=1$ with the same input rate as in Figs. 7(a)7 (c), respectively. In the case of $K=1$, if the input rate is equal to $1 / 1.1$, the second eigenvalue of the corresponding Markov operator shows that the response is outside of $1: 1$ stochastic phase locking [Fig. 7(d)]. The peak of the ISI density in Fig. 7(d) had a sharper peak than the one in Fig. 7(a). As the input rate decreased, the peak shifted to larger ISI values as in the case of $K=\infty$ and the magnitude decreased rapidly than that of $K=\infty$. The appearance of the plateau and three local maxima in the ISI density was also different, i.e., the shorter plateau in Fig. 7(e) and the magnitude of the local maxima
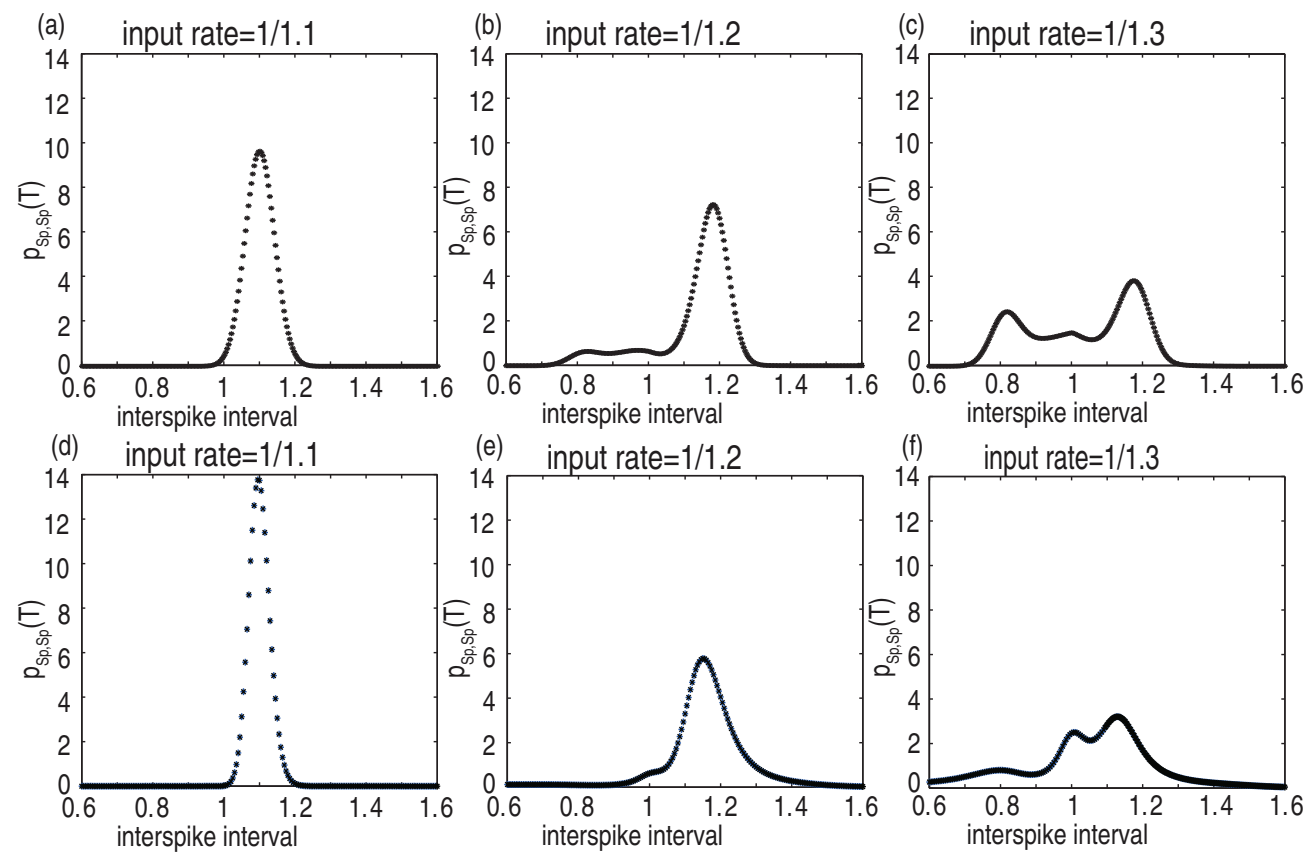

FIG. 7. Interspike interval density. Interspike interval densities of the reduced model calculated using Eq. (56) $[K=\infty$, (a), (b), and (c)] and the full model by solving Eq. (6) using Euler-Maruyama method $[K=1$, (d), (e), and (f)], respectively. (a) The interspike interval density in the $1: 1$ stochastic phase locking region. Panels (b) and (c) show the densities outside the $1: 1$ stochastic phase locking region for $I=1.2$ and $I=1.3$, respectively. There is a stochastic bifurcation point between (a) and (b), but the interspike interval density changed smoothly because the invariant density of the corresponding SPTO changed smoothly. In the case of $K=1$, there is not a stochastic bifurcation point between (d) and (e), there is a stochastic bifurcation point lower than $I=1$.1. Plots are shown for $A=0.95$ and $\epsilon=0.3$. Input rate is shown in each plot. The abscissa and ordinate are interspike interval and probability density, respectively. 
decreased as the ISI decreased in Fig. 7(f). In the case of $K=\infty$, the accuracy of the calculated ISI density depended on the input rate, and especially for input rates higher than 1 , calculating the ISI was difficult within the scope of the reduced model, one reason being that the convergence of the summation $\Sigma_{j=1}^{\infty} p_{S p, I m}(\tau)$ in Eq. (40) was slow.

\section{F. Dependence of the current instantaneous stochastic rotation number on the past activity of the stochastic Poincaré oscillator}

To understand the effects of the past activity of the stochastic Poincaré oscillator on the current instantaneous stochastic rotation number, we calculated the past components that determine the current instantaneous stochastic rotation number. As an example, let us consider the instantaneous stochastic rotation numbers corresponding to five impulses with a fixed impulse amplitude $A$ and four interimpulse intervals denoted by $I_{1}, I_{2}, I_{3}$, and $I_{4}$. The equation that determines the instantaneous stochastic rotation number at the last interimpulse interval is

$$
\begin{aligned}
\Omega_{K, \epsilon, A, I_{4}} & \int_{0}^{1} \int_{0}^{\infty} w_{K, \epsilon, A, I_{4}}(r, \phi) h_{K, \epsilon, A, I_{3}}^{*}(r, \phi) d r d \phi \\
& +\int_{0}^{1} \int_{0}^{\infty} w_{K, \epsilon, A, I_{4}}(r, \phi) \mathcal{Q}_{K, \epsilon, A, I_{3}} h_{K, \epsilon, A, I_{2}}^{*}(r, \phi) d r d \phi \\
& +\int_{0}^{1} \int_{0}^{\infty} w_{K, \epsilon, A, I_{4}}(r, \phi) \mathcal{Q}_{K, \epsilon, A, I_{3}} \mathcal{Q}_{K, \epsilon, A, I_{2}} \\
& \times h_{K, \epsilon, A, I_{1}}^{*}(r, \phi) d r d \phi \\
& +\int_{0}^{1} \int_{0}^{\infty} w_{K, \epsilon, A, I_{4}}(r, \phi) \mathcal{Q}_{K, \epsilon, A, I_{3}} \mathcal{Q}_{K, \epsilon, A, I_{2}} \mathcal{Q}_{K, \epsilon, A, I_{1}} \\
& \times h_{1}(r, \phi) d r d \phi .
\end{aligned}
$$

The first term in Eq. (59) is determined by the invariant density that corresponds to the third impulse and the mean lifted angular coordinate difference at the fourth impulse. Thus, the first term depends on $I_{3}$ and $I_{4}$. We assign $I_{3}$, that is, the oldest interimpulse interval in this term, to this term to show that it depends on the past activity from the third impulse. Similarly, the second term of Eq. (59) depends on $I_{2}, I_{3}$, and $I_{4}$, and we assign $I_{2}$ to the second term. The sum of the third and fourth terms of Eq. (59) depends on $I_{1}, I_{2}, I_{3}$, and $I_{4}$, and we assign $I_{1}$ to the sum of these terms. This correspondence shows the dependence of the current instantaneous stochastic rotation number on the past activity of the stochastic Poincaré oscillator, and this can also be seen in Fig. 8 as a function of the input rate.

The components of the instantaneous stochastic rotation number in response to impulses with a fixed amplitude are shown in Fig. 8 (the components are denoted by asterisks, and the filled square and open square show the current instantaneous stochastic rotation number). Figures 8(a) and 8(b) use the same set of input rates but different signs of $\left|f_{\text {step }}\right|$, i.e., the input rates change in opposite directions. The difference in the sign of $f_{\text {step }}$ induced a different dependence of the current instantaneous stochastic rotation number on the past activity of the stochastic Poincaré oscillator: Figure 8(a)
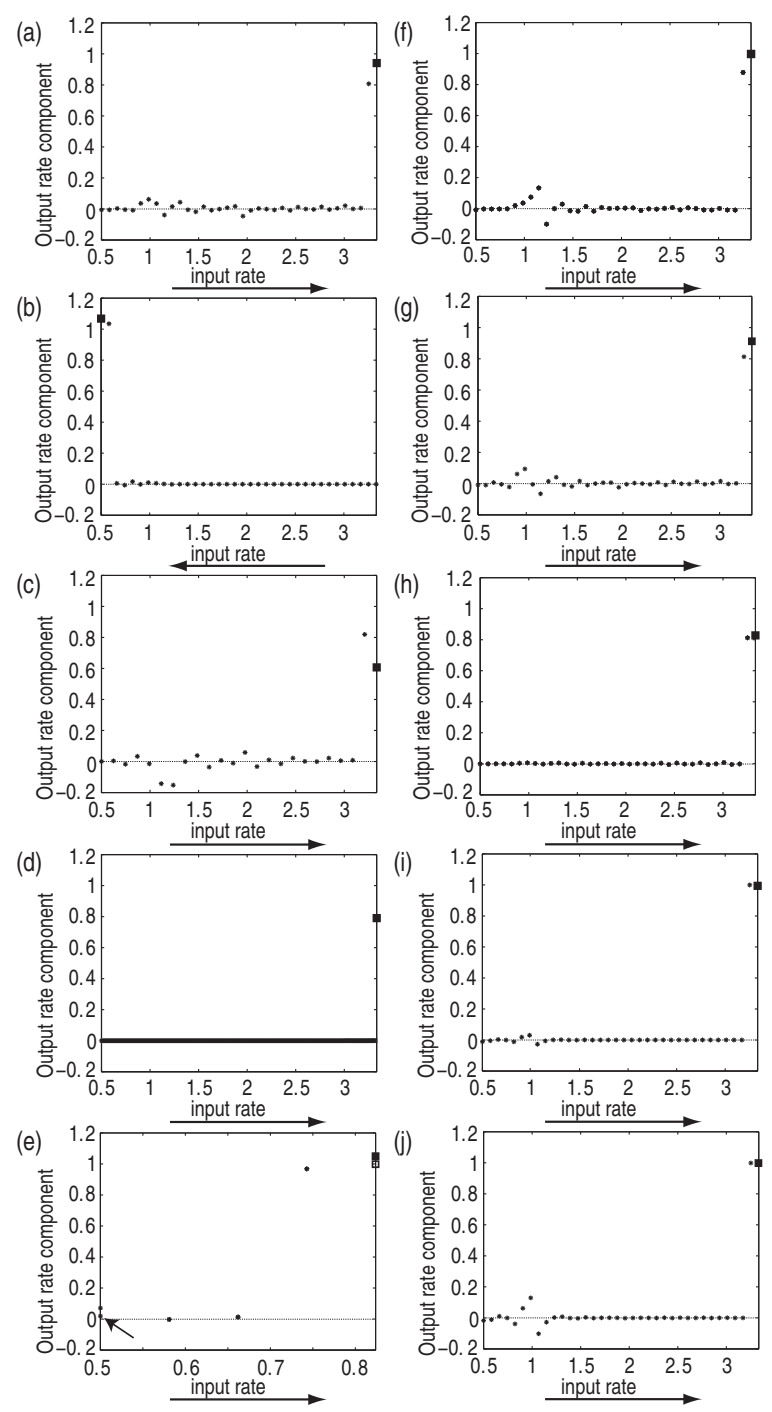

FIG. 8. Dependence of the current instantaneous stochastic rotation number on the past activity of the stochastic Poincare oscillator. Each component of the current instantaneous stochastic rotation number explained in Eq. (59) is plotted as a function of the input rates. The filled squares [filled square and open square in (e)] show the current instantaneous rotation number in each panel. (a) Standard plot for starting and final input rates of $f_{\text {start }}=0.5$ and $f_{\text {end }}=1 / 0.3$, $N=35$ impulses with an amplitude of $A=0.95$, a relaxation rate of $K=1$, and a noise strength of $\epsilon=0.3$. The initial density $h_{1}\left(r_{1}, \phi_{1}\right)$ was a uniform distribution with a support of $\left(r_{1}, \phi_{1}\right) \in(0,3.5] \times[0,1)$. In (b)-(j), the effects of various parameters are calculated by varying one parameter while the other parameters and initial density are the same as those in (a). The output components as a function of the input rate for (b) $f_{\text {start }}=1 / 0.3$ and $f_{\text {end }}=0.5$ to investigate the effect of $f_{\text {step }}$ in Eq. (58), (c) $N=23$, (d) $N=500$, (there is an overlap of the filled square and the asterisk), and (e) $f_{\text {start }}=0.5$, $f_{\text {end }}=0.82381$, and $N=4$. In (e) results for two initial densities are shown: the density in (a) (asterisks) and a uniform distribution with a support of $\left(r_{1}, \phi_{1}\right) \in(0,3.5] \times[0.25,0.75)$ (asterisks indicated by an arrow). There are three overlaps between two responses, and the filled square and open square show the corresponding current instantaneous rates. Plots are also shown for (f) $K=\infty$, (g) $K=0.6$, (h) $\epsilon=0.6$, (i) $A=0.25$, and (j) $A=0.45$. In each panel, arrow under the label "input rate" indicates the direction of the input rate and dashed line indicates zero level of the output rate component. 
shows a relatively larger component value for input rates between $\sim 1.0$ and 2.0 and a weaker dependence on impulses near the current input rate is seen, except for the component just before the final input rate at which the current output rate is shown. In contrast, Fig. 8(b) shows that the instantaneous stochastic rotation number depended only on components near the current input rate.

As the number of the impulses increased, the variation in the components tended to decrease [compare Fig. 8(a) with Figs. 8(c) and 8(d)]. Figure 8(d) shows the components of the instantaneous stochastic rotation number corresponding to Fig. 6(e). As the number of impulses determined by Eq. (57) increases, the difference between adjacent invariant densities corresponds to the input rate, and components that depend on the initial density decrease in magnitude. Thus, the contribution of components, apart from the component just before the current input rate, subsequently decrease.

The instantaneous stochastic rotation number and components at the fifth impulse are shown in Fig. 8(e) for two different initial densities [the filled square and asterisks corresponds to the case in Fig. 6(a) and the open square and asterisks to that in Fig. 6(c)]. For some input rates, the components for the two cases are superimposed. This difference would appear to originate from the difference in the initial densities.

Furthermore, we checked the dependence of the current instantaneous stochastic rotation number on the past activity by changing the relaxation rate [Figs. $8(\mathrm{f})$ and $8(\mathrm{~g})$ ], the noise strength [Fig. 8(h)], and the impulse amplitude [Figs. 8(i) and $8(\mathrm{j})$ ], respectively. For the finite relaxation rate, an increase of the relaxation rate led to the lower variation of the past components except the components corresponding to the 17th-20th impulses [compare Fig. 8(a) with Fig. 8(g)]. However, for the infinite relaxation rate [compare Fig. 8(a) and Fig. 8(f)], the variation of the components from the 8th impulse to the 16th impulse was larger than that for the finite relaxation rate and that of the components corresponding to the 17th-20th impulses decreased. An increase in the noise strength induced that, as can be seen in a comparison of Figs. 8(a) and 8(h), a larger noise strength induced a smaller variation in the components. As the impulse amplitude increased until about 0.45 [compare Figs. 8(i) and 8(j)], the variability of the past components around the input rate 1 increased. If the impulse amplitude increased beyond about 0.45 [compare Figs. 8(a) and 8(j)], the overall trend of the variability around the input rate 1 decreased, and the variability of some components after the ninth impulse increased slightly.

\section{DISCUSSION}

The transient regime should be short enough to encode information in the spike pattern in nervous systems. The length of the transient regime becomes an indicator of the extent of the dependence on the past neural activity. By decomposing the current instantaneous stochastic rotation number into the past activity components, we were able to tackle this problem. The results showed that the components of the current instantaneous stochastic rotation number can be negative, and components far from the current input rate can affect the current instantaneous stochastic rotation number. Some components depend on the difference between the invariant densities of adjacent discretized SPTOs in the product of discretized SPTOs, which determines the density in the equation of the current instantaneous stochastic rotation number. A larger difference between invariant densities may increase the values of the corresponding components. Furthermore, the product of discretized SPTOs shows weak ergodicity, that is, the stochastic Poincaré oscillator can forget the initial density, and equally, the current instantaneous stochastic rotation number can forget the initial density or initial condition. This situation may arise in nervous systems if a neuron receives a sufficient number of impulses.

The kernel density estimation is a method to estimate spike rate [49-54]. In this method, the spike train is convoluted with a kernel function to estimate the spike rate. Further analysis of the past activity components of the current instantaneous stochastic rotation number might lead to an adequate selection of the kernel function, that is usually a non-negative function, and its width to calculate the spike rate.

As shown by an examination of the stochastic rotation number, the steady-state and transient responses can be different. In a related experiment it was found that the response of a pacemaker neuron in crayfish was different depending on the past inputs [55-57]. We used the definition of the instantaneous stochastic rotation number as given by Eqs. (32) and (36), since we examined the input-output firing rate relationship as shown in Fig. 6. This is a natural extension of the stochastic rotation number in the steady state to that in the transient state. This is also required in experiments to establish the input-output rate relationship. In fact, past studies typically relate the input and output rates empirically (see $[55,56]$ for examples). To define the SPTO and instantaneous stochastic rotation number for a continuous input over a continuous time period leads to the firing rate over that continuous time period, and this is a topic for future study. Analysis using the continuous-time version of the SPTO will yield the continuous dependence of the current firing rate on the past activity of the neuron model and give some insight into information coding in nervous systems.

For the model dynamics, we showed that the contribution of the past activity of the stochastic Poincaré oscillator to the current density was defined by Eq. (26). The product of the discretized SPTOs determines the response, i.e., the current density, of the neuronal oscillator to time-varying impulses. Alternatively, the effect of the terms in Eq. (26) can be examined by using the one-norm, if necessary, and these terms show the dependence of the current density on the past neuronal activity.

A shorter dependence on the past activity is necessary for carrying information via a spike pattern, and a longer dependence on the past activity might lead to information being carried with a weighted average of the number of spikes that is determined by the transient dynamics of the neuron. To understand the mechanism behind this dependence, it is necessary to understand the spectral structure of the discretized SPTO. A response of the stochastic Poincaré oscillator, or a product of the discretized SPTOs, depends on the discretized SPTOs selected by the input parameters. Hence, it is an important problem to understand how each discretized SPTO in a product of discretized SPTOs affects the 
spectral property of the product. Since the discretized SPTO is a noncommutative matrix, the order of the multiplication also affects this spectral property.

As mentioned in the Introduction, we focused on the transient dynamics of a neuronal oscillator. Usually, conventional statistics such as the spike train power spectrum, Fano factor of the spike count, and ISI density assume that the oscillator is in the steady state. These statistics are not adequate for quantifying the transient dynamics of the neuronal oscillator. Furthermore, it is difficult to derive the evolution of the transient ISI density from the current setting of the stochastic Poincaré oscillator, and thus we did not calculate the interspike interval serial correlation coefficient analytically. Instead, we introduced the instantaneous stochastic rotation number in this study and analyzed the corresponding components. In this way, one can quantify the effect of the past activity of neuronal oscillators. It is also a topic for future study to derive the transient counterparts of the conventional statistics.

Equations (38) and (39) offer a way of determining the components of the current instantaneous stochastic rotation number experimentally. As an example, consider the instantaneous stochastic rotation numbers corresponding to five impulses with fixed impulse amplitudes, i.e., the impulses are characterized by four interimpulse intervals $I_{1}, I_{2}, I_{3}$, and $I_{4}$ [Fig. 9 and Eq. (59)]. Consider first the instantaneous stochastic rotation number corresponding to the last interimpulse interval. The equation that determines the stochastic

(a)

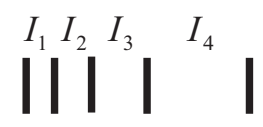

(b) || $\mid$

(c)

(d)

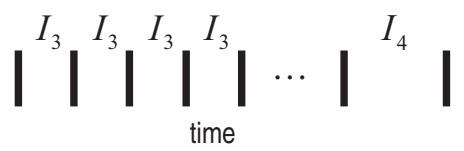

FIG. 9. Experimental procedure to determine the components of the current instantaneous stochastic rotation number. (a) Five impulses determined by a constant impulse amplitude $A$ and four interimpulse intervals of $I_{1}, I_{2}, I_{3}$, and $I_{4}$ are added repeatedly to a spontaneously firing neuron with a recovery period. We decompose the current firing rate, i.e., the firing rate during $I_{4}$, into the components determined by the past activity of the neuron. Impulses to measure (b) the summation of the first, second, and third terms, (c) the summation of the first and second terms, and (d) the first term in Eq. (59). The firing rate during $I_{4}$ needs to be measured by repeatedly adding the impulses to the neuron with a recovery period. Subtraction using the measured firing rates during $I_{4}$ in (a)-(d) leads to the decomposition of the firing rate during $I_{4}$ in (a) into the components in Eq. (59). In all panels, abscissa is time. See the discussion for a detailed explanation. rotation number at the last interimpulse interval is Eq. (59), and to begin, we add five impulses with interimpulse intervals of $I_{1}, I_{2}, I_{3}$, and $I_{4}$ and an intertrial interval repeatedly to a spontaneously firing neuron with some noise to measure the firing rate during the interimpulse interval $I_{4}, \Omega_{K, \epsilon, A, I_{4}}$ [Fig. 9(a)]. After a recovery period, we add a sufficient number of impulses with an interimpulse interval of $I_{1}$ to the neuron to achieve the corresponding steady state $h_{K, \epsilon, A, I_{1}}^{*}$ and then add four impulses with interimpulse intervals $I_{2}$, $I_{3}$, and $I_{4}$ and an intertrial interval to the same neuron [Fig. 9(b)]. After repeating this procedure, the measured firing rate during the interimpulse interval $I_{4}\left(\Omega_{K, \epsilon, A, I_{4}, b}\right)$ in Fig. 9(b) is equal to the summation of the first, second, and third terms in Eq. (59), since $h_{1}(r, \phi)=h_{K, \epsilon, A, I_{1}}^{*}$, and this leads to $\mathcal{Q}_{K, \epsilon, A, I_{1}} h_{1}(r, \phi)=0$ [see the definition of $\mathcal{Q}_{K, \epsilon, A, I}$ in Eq. (23)]. That is, the fourth component in Eq. (59) is equal to zero. Thus, $\Omega_{K, \epsilon, A, I_{4}}-\Omega_{K, \epsilon, A, I_{4}, b}$ is equal to the fourth term in Eq. (59). After a recovery period, we then add a sufficient number of impulses with an interval $I_{2}$ to the same neuron to achieve the corresponding steady state $h_{K, \epsilon, A, I_{2}}^{*}$ and then add three impulses with interimpulse intervals of $I_{3}$ and $I_{4}$ and an intertrial interval. After repeating this stimulation, the firing rate during the interimpulse interval $I_{4}\left(\Omega_{K, \epsilon, A, I_{4}, c}\right)$ in Fig. 9(c) is equal to the summation of the first and second terms in Eq. (59). Thus, $\Omega_{K, \epsilon, A, I_{4}, b}-\Omega_{K, \epsilon, A, I_{4}, c}$ is equal to the third term in Eq. (59). Again, after a recovery period, impulses with an interval $I_{3}$ are added to the same neuron to achieve the corresponding steady state $h_{K, \epsilon, A, I_{3}}^{*}$, and two impulses with an interimpulse interval of $I_{4}$ are added to the same neuron. After repeating this stimulation with an intertrial interval, the firing rate during $I_{4}\left(\Omega_{K, \epsilon, A, I_{4}, d}\right)$ in Fig. $9(\mathrm{~d})$ is equal to the first term in Eq. (59). Thus, $\Omega_{K, \epsilon, A, I_{4}, c}-$ $\Omega_{K, \epsilon, A, I_{4}, d}$ is equal to the second term in Eq. (59). In this way, one can experimentally decompose the current instantaneous stochastic rotation number. The intrinsic noise strength of a neuron depends on the number of ion channels. The dynamicclamp technique is one possible way to change the channel noise experimentally [58]. Combining this technique and the above-mentioned stimulus makes it possible to decompose the current instantaneous stochastic rotation number and examine the effect of the intrinsic noise strength on the decomposition.

An increase in the noise strength smoothed and decreased the variation in the components of the instantaneous stochastic rotation number, which means that the dependence on the instantaneous stochastic rotation number may decrease as the noise strength increases. However, temporal coding is not possible in this case because the larger noise makes the firing time inaccurate. Furthermore, the experimental results of Perkel et al. [59] revealed phase locking in pacemaker neurons, and to consider the larger impulse amplitude, it is necessary to understand the global picture of the response of spontaneously firing neurons.

In terms of the dynamics of a spontaneously firing neuron in response to time-varying impulses, it is necessary to investigate the statistical behavior of the neuron in response to impulses with a constant amplitude and interimpulse interval. Thus, it is necessary to develop a method of constructing the SPTO or its analog experimentally. It appears to be clear that if a spontaneously firing neuron is modeled by stochastic 
differential equations, this problem reduces to constructing the SPTO using the given equations. The construction of the SPTO from stochastic differential equations is not model dependent, since the small disturbance asymptotic theory is general. Thus, one can construct the SPTO for other impulse-driven biological or nonlinear oscillators.

Finally, for the case in which the instantaneous stochastic rotation number depends on the past activity, it is difficult to encode information in the spike pattern since the spike generation also depends on the past activity. Our method can evaluate the dependence of the current state of a neuronal oscillator on the past activity. The amount of the past activity depended on the input parameters, the relaxation rate, and noise strength. To evaluate the past dependency of various neurons and their models using our method or its extension would offer a way to gain insight into the information carrier in nervous systems.

\section{ACKNOWLEDGMENTS}

The author thanks Professor A. Takahashi for providing preprints. The author also thanks the anonymous referees for the careful reading and the valuable comments and suggestions to improve this paper. This work was supported by the Japan
Science and Technology Agency, Precursory Research for Embryonic Science and Technology Program.

\section{APPENDIX}

The considered model may not be general enough. However, it has been used as an important standard model and is enough for the purpose in this paper. First of all, analyses using the Markov operators are also applicable to neuronal oscillators described by stochastic differential equation with some regularity conditions of the drift and diffusion coefficients. Second, as pointed out by Izhikevich [9], "When a neuronal model is far from the subcritical Andronov-Hopf bifurcation, its phase portrait may look similar to the one corresponding to the supercritical Andronov-Hopf bifurcation." The Stuart-Landau oscillator is the normal form of a supercritical Andronov-Hopf bifurcation and can be transformed to the Poincaré oscillator $[16,60]$. Moreover, it is shown that the modified Poincaré oscillators with slow and fast dynamics reproduce the response of pacemaker neurons, which can be transformed to the Poincaré oscillator [4,22]. In these transformations, the effect of the relaxation dynamics of the neural oscillators with refractory period can be put into the perturbation term.
[1] F. Rieke, D. Warland, R. de de Ruyter van Steveninck, and W. Bialek, Spikes: Exploring the Neural Code (Computational Neuroscience) (Bradford, Cambridge, MA, 1999).

[2] J. P. Segundo, M. Stiber, E. Altshuler, and J. F. Vibert, Neuroscience 62, 459 (1994).

[3] M. Stiber, R. Ieong, and J. Segundo, IEEE T. Neural Networks 8, 1379 (1997).

[4] T. Yamanobe, K. Pakdaman, T. Nomura, and S. Sato, Biosystems 48, 287 (1998).

[5] T. Yamanobe, Phys. Rev. E 84, 011924 (2011); 88, 019904 (2013).

[6] M. I. Rabinovich, P. Varona, A. I. Selverston, and H. D. Abarbanel, Rev. Mod. Phys. 78, 1213 (2006).

[7] M. Rabinovich, R. Huerta, and G. Laurent, Science 321, 48 (2008).

[8] F. C. Hoppensteadt and E. M. Izhikevich, Weakly Connected Neural Networks (Applied Mathematical Sciences) (Springer, New York, 1997).

[9] E. M. Izhikevich, Dynamical Systems in Neuroscience: The Geometry of Excitability and Bursting (MIT, Cambridge, MA, 2010).

[10] M. R. Guevara, L. Glass, and A. Shrier, Science 214, 1350 (1981).

[11] L. Glass, M. R. Guevara, A. Shrier, and R. Perez, Physica D 7, 89 (1983).

[12] J. C. Smith, H. H. Ellenberger, K. Ballanyi, D. W. Richter, and J. L. Feldman, Science 254, 726 (1991).

[13] S. M. Johnson, J. C. Smith, G. D. Funk, and J. L. Feldman, J. Neurophysiol. 72, 2598 (1994).

[14] K. Wiesenfeld, P. Colet, and S. H. Strogatz, Phys. Rev. E 57, 1563 (1998).

[15] F.-F. Jin, J. Atmos. Sci. 54, 811 (1997).
[16] Y. Kuramoto, Chemical Oscillations, Waves, and Turbulence (Dover Books on Chemistry) (Dover, New York, 2003).

[17] A. T. Winfree, The Geometry of Biological Time, 2nd ed. (Springer-Verlag, New York, 2000).

[18] F. C. Hoppensteadt and J. P. Keener, J. Math. Biol. 15, 339 (1982).

[19] L. Glass and M. C. Mackey, From Clocks to Chaos (Princeton University Press, Princeton, NJ, 1988).

[20] L. Glass and J. Sun, Phys. Rev. E 50, 5077 (1994).

[21] M. Stiber, K. Pakdaman, J. F. Vibert, E. Boussard, J. P. Segundo, T. Nomura, S. Sato, and S. Doi, Biosystems 40, 177 (1997).

[22] T. Nomura, S. Sato, S. Doi, J. P. Segundo, and M. D. Stiber, Biol. Cybern. 72, 93 (1994).

[23] T. Yamanobe and K. Pakdaman, Biol. Cybern. 86, 155 (2002).

[24] K. Pakdaman and D. Mestivier, Phys. Rev. E 64, 030901 (2001).

[25] H. Croisier, M. R. Guevara, and P. C. Dauby, Phys. Rev. E 79, 016209 (2009).

[26] J. A. White, J. T. Rubinstein, and A. R. Kay, Trends Neurosci. 23, 131 (2000).

[27] N. Kunitomo and A. Takahashi, Ann. Appl. Probab. 13, 914 (2003).

[28] A. Takahashi, K. Takehara, and M. Toda, Int. J. Theor. Appl. Finan. 15, 1250044 (2012).

[29] M. R. Guevara and L. Glass, J. Math. Biol. 14, 1 (1982).

[30] J. P. Keener and L. Glass, J. Math. Biol. 21, 175 (1984).

[31] L. Glass, Nature (London) 410, 277 (2001).

[32] T. Tateno and H. P. C. Robinson, Biophys. J. 92, 683 (2007).

[33] K. Yoshimura and K. Arai, Phys. Rev. Lett. 101, 154101 (2008).

[34] F. R. Gantmacher, The Theory of Matrices (Chelsea, New York, 1959), Vol. II. 
[35] A. Lasota and M. C. Mackey, Chaos, Fractals, and Noise: Stochastic Aspects of Dynamics (Applied Mathematical Sciences) (Springer, New York, 1994).

[36] J. Ding and A. Zhou, Nonnegative Matrices, Positive Operators, and Applications (World Scientific, New Jersey, 2009).

[37] S. Doi, J. Inoue, and S. Kumagai, J. Stat. Phys. 90, 1107 (1998).

[38] T. Tateno, S. Doi, S. Sato, and L. M. Ricciardi, J. Stat. Phys. 78, 917 (1995).

[39] T. Tateno, J. Stat. Phys. 92, 675 (1998).

[40] J. Inoue, S. Doi, and S. Kumagai, Phys. Rev. E 64, 056219 (2001).

[41] T. Tateno, Phys. Rev. E 65, 021901 (2002).

[42] L. Arnold, Random Dynamical Systems (Springer Monographs in Mathematics) (Springer, Berlin, 2008).

[43] A. Borisyuk and F. Rassoul-Agha [SIAM Appl. Dyn. Syst. (to be published)].

[44] E. Seneta, Non-negative Matrices and Markov Chains (Springer Series in Statistics) (Springer, New York, 2006).

[45] I. C. Ipsen and T. M. Selee, SIAM J. Matrix Anal. A 32, 153 (2011).

[46] W. H. Nesse, G. A. Clark, and P. C. Bressloff, Phys. Rev. E 75, 031912 (2007).

[47] See Supplemental Material at http://link.aps.org/supplemental/ 10.1103/PhysRevE.88.052709 for video S1 (six movies showing the evolution of the density and corresponding transient com- ponents for $K=0.25,1$, and $\infty$ using the parameters given in Fig. 3) and video $S 2$ (dependence of the invariant density on the input rate for $K=1, \epsilon=0.3$, and $A=0.95$ ).

[48] T. Tateno and Y. Jimbo, Phys. Lett. A271, 227 (2000).

[49] E. Parzen, Ann. Math. Stat. 33, 1065 (1962).

[50] M. Rosenblatt, Ann. Math. Stat. 27, 832 (1956).

[51] A. C. Sanderson, IEEE T. Biomed. Eng. 27, 271 (1980).

[52] B. J. Richmond, L. M. Optican, and H. Spitzer, J. Neurophysiol. 64, 351 (1990).

[53] M. Nawrot, A. Aertsen, and S. Rotter, J. Neurosci. Methods 94, 81 (1999)

[54] H. Shimazaki and S. Shinomoto, J. Comput. Neurosci. 29, 171 (2009).

[55] J. P. Segundo, J. F. Vibert, M. Stiber, and S. Hanneton, Neuroscience 68, 657 (1995).

[56] J. P. Segundo, M. Stiber, J. F. Vibert, and S. Hanneton, Neuroscience 68, 693 (1995).

[57] J. P. Segundo, J. F. Vibert, and M. Stiber, Neuroscience 87, 15 (1998).

[58] A. D. Dorval, Jr. and J. A. White, J. Neurosci. 25, 10025 (2005).

[59] D. H. Perkel, J. H. Schulman, T. H. Bullock, G. P. Moore, and J. P. Segundo, Science 145, 61 (1964)

[60] Y. Kuznetsov, Elements of Applied Bifurcation Theory (Applied Mathematical Sciences), 3rd ed. (Springer, New York, 2004). 